\title{
Weak imposition of essential boundary conditions in the finite element approximation of elliptic problems with non-matching meshes
}

\author{
Ramon Codina and Joan Baiges \\ Centre Internacional de Mètodes Numèrics en Enginyeria, \\ Universitat Politècnica de Catalunya, \\ Jordi Girona 1-3, Edifici C1, 08034 Barcelona, Spain. \\ ramon.codina@upc.edu,jbaiges@cimne.upc.edu
}

\section{Contents}

1 Introduction $\quad 2$

2 Description of the method for an abstract problem 4

3 Poisson's problem $\quad 6$

4 Darcy's problem $\quad \mathbf{8}$

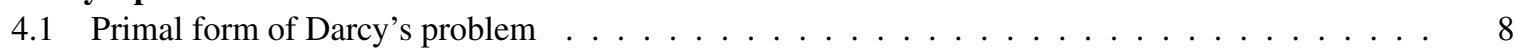

4.1.1 Galerkin finite element approximation . . . . . . . . . . . . . . . . . . . 8

4.1.2 Stabilized finite element approximation . . . . . . . . . . . . . . . . . 10

4.1 .3 Matrix structure . . . . . . . . . . . . . . . . . . 11

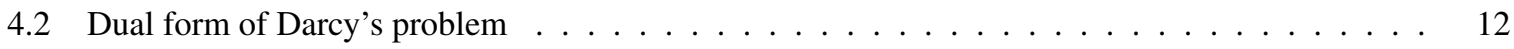

4.2.1 Galerkin finite element approximation . . . . . . . . . . . . . . . . . . . . . . . . . . . . . . . . .

4.2.2 Stabilized finite element approximation . . . . . . . . . . . . . . . . . . . 15

4.2.3 Matrix structure ........................ 16

5 Stokes' problem $\quad 18$

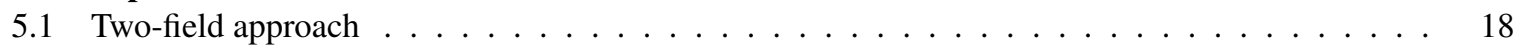

5.1 .1 Galerkin finite element approximation . . . . . . . . . . . . . . . 18

5.1 .2 Stabilized finite element approximation . . . . . . . . . . . . . . . . . 20

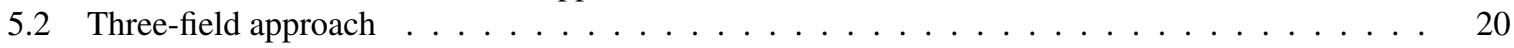

5.2.1 Galerkin finite element approximation . . . . . . . . . . . . . . . . . . . 21

5.2.2 Stabilized finite element approximation . . . . . . . . . . . . . . . 22

6 Numerical examples $\quad 2$

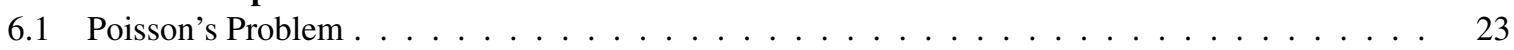

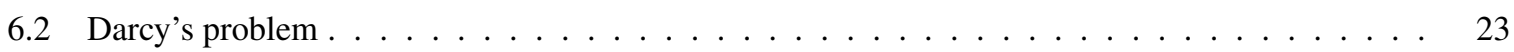

6.3 Stokes'Problem . . . . . . . . . . . . . . . . . . . . . . . . 24

$\begin{array}{llr}7 & \text { Conclusions } & 28\end{array}$ 


\begin{abstract}
In this work we propose a method to prescribe essential boundary conditions in the finite element approximation of elliptic problems when the boundary of the computational domain does not match with the element boundaries. The problems considered are the Poisson problem, the Stokes problem and the Darcy problem, the latter both in the primal and in the dual formulation. The formulation proposed is of variational type. The key idea is to start with the variational form that defines the problem and treat the boundary condition as a constraint. The particular feature is that the Lagrange multiplier is not defined on the boundary where the essential condition needs to be prescribed, but is taken as a certain trace of a field defined in the computational domain, either in all of it or just in a region surrounding the boundary. When approximated numerically, this may allow one to condense the degrees of freedom of the new field and end up with a problem posed only in terms of the original unknowns. The nature of the field used to weakly impose boundary conditions depends on the problem being treated. For the Poisson problem it is a flux, for the Stokes problem a stress, for the Darcy problem in primal form a velocity field and for the Darcy problem in dual form it is a potential. If this new variable is eliminated, the resulting problem resembles Nitsche's way to impose boundary conditions, with the advantage that no conditions on the parameter are required for stability and, in particular, that this parameter can be taken smaller in the formulation we propose.
\end{abstract}

\title{
1 Introduction
}

Non-matching mesh (or grid) methods are numerical approximations to partial differential equations in which the discretization of the computational domain does not match its boundary (see for example the review in [9]). The interest is obvious: mesh generation is greatly simplified and it is easy to deal with domain motion when it occurs. Moreover, when Cartesian grids are used, the numerical approximation itself can also be simplified, using for example simple and efficient finite difference schemes. However, and in order to fix ideas, we will assume throughout that the finite element method is used as numerical formulation.

The problem of having the computational domain embedded in a mesh is the prescription of boundary conditions, particularly when these are of essential or Dirichlet type, that is to say, the unknown of the problem itself must be equal to a given datum. Let us describe the problem to be solved. Consider the situation depicted in Fig. 1. A domain $\Omega \subset \mathbb{R}^{d}, d=2,3$, with boundary $\Gamma=\partial \Omega$ (red curve in Fig. 1), is covered by a mesh that occupies a domain $\Omega_{h}=\Omega_{\text {in }} \cup \Omega_{\Gamma}$, where $\Omega_{\text {in }} \subset \Omega$ is formed by the elements interior to $\Omega$ and $\Omega_{\Gamma}$ is formed by a set of elements cut by $\Gamma$. In turn, let us split $\Omega_{\Gamma}=\Omega_{\Gamma \text {,in }} \cup \Omega_{\Gamma \text {,out }}$, where $\Omega_{\Gamma \text {,in }}=\Omega \cap \Omega_{\Gamma}$ and $\Omega_{\Gamma \text {,out }}$ is the interior of $\Omega_{\Gamma} \backslash \Omega_{\Gamma, \text { in }}$. Note that $\Omega=\Omega_{\text {in }} \cup \Omega_{\Gamma, \text { in }}$.

Suppose we want to solve a boundary value problem for the unknown $u$ in $\Omega$ with the mesh of $\Omega_{h}$ already created and boundary conditions $u=\bar{u}$ on $\Gamma$. The obvious choice would be:

- Obtain the nodes of $\Gamma$ (circles in Fig. 1) from the intersection with the element edges.

- Split the elements of $\Omega_{\Gamma, \text { in }}$ so as to obtain a grid matching the boundary $\Gamma$.

- Prescribe the boundary condition $u_{h}=\bar{u}$ in the classical way, where $u_{h}$ denotes the approximate solution.

This strategy leads to a local remeshing close to $\Gamma$ that is involved from the computational point of view. Obviously, the implementation of the strategy described is very simple for unstructured simplicial meshes, but it is not so easy if one wants to use other element shapes and, definitely, prevents from using Cartesian meshes. Moreover, if the boundary $\Gamma$ evolves in time the number of degrees of freedom changes at each time instant, thus modifying the structure and sparsivity of the matrix of the final algebraic system.

The time evolution of the computational domain is in fact a paradigmatic situation for the use of non-matching meshes, since they are required if one wants to use a fixed mesh during all the calculation. In the classical Arbitrary Lagrangian-Eulerian (ALE) approach to solve problems in computational fluid dynamics, the mesh in which the computational domain is discretized is deformed (see for example [13]). This is done according to a prescribed motion of part of its boundary, which is transmitted to the interior nodes in a way as smooth as possible so as to avoid mesh distortion. The Fixed-Mesh-ALE (FM-ALE) formulation proposed in [11] has a different motivation. Instead of assuming that the computational domain is defined by the mesh boundary, we 


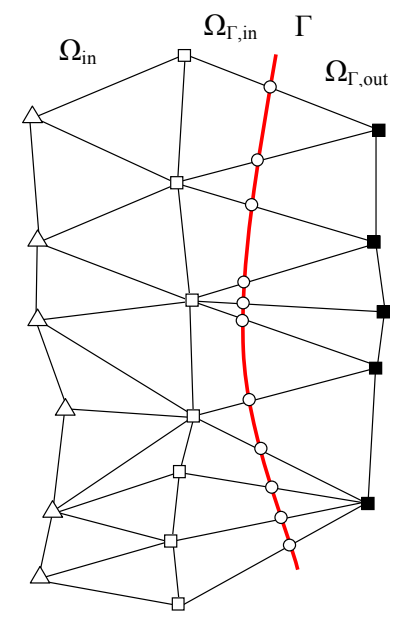

Figure 1: Problem setting

assume that there is a function that defines the boundary of the domain where the flow takes place. It may be given, for example, by the shape of a body that moves within the fluid, or it may need to be computed, as in the case of level set functions. It may be also defined discretely, by a set of points. When this boundary function moves, the flow domain changes, and that must be taken into account at the moment of writing the conservation equations that govern the flow, which need to be cast in the ALE format. If essential (Dirichlet) boundary conditions need to be prescribed on the moving boundaries, this prescription must account for the fact that the mesh will not match these boundaries.

Other possibilities to use a single grid in the whole simulation can be found in the literature, each one having advantages and drawbacks. They were designed as an alternative to body fitted meshes and can be divided into two main groups, corresponding in fact to two ways of prescribing the boundary conditions on the moving boundary [10]:

- Introduction of a force term. The boundary conditions are taken into account through a force term, which appears either in the strong or in the weak form of the equations. Among this type of methods, let us cite for example the Immersed Boundary method as a variant of the Penalty method, where punctual forces are added to the momentum equation, and the Fictitious Domain method, where the solid boundary conditions are imposed through a Lagrange multiplier.

- Approximate boundary conditions. Instead of adding a force term, these methods impose the boundary conditions in an approximate way once the discretization has been carried out, either by modifying the differential operators near the interface (in finite differences) or by modifying the unknowns near the interface.

The Immersed Boundary Method (IBM) in its original form [23] consists in adding punctual penalty forces computed from physical models (elastic membranes, in the original reference) in the domain boundary, so that the boundary conditions are fulfilled. The method is first order accurate even if second order approximation schemes are used, although formal second order accuracy has been reported in [19]. The more recent Immersed Interface Method achieves higher order accuracy by avoiding the use of the Dirac delta distribution to define the forcing terms (see [20,21, 25]). The Penalty method is similar to the previous one in the sense that a force term is added to the momentum equations. The difference is due to the fact that the penalty parameter is simply required to be large enough to enforce the boundary conditions approximately [24], and not computed based on physical grounds as the IBM.

Another approach is the use of Lagrange multipliers to enforce the boundary conditions. However, the finite element subspaces for the bulk and Lagrange multiplier fields must satisfy the classical inf-sup condition, which usually leads to the need for stabilization. Moreover, additional degrees of freedom must be added to the problem. The use of Lagrange multipliers is the basis of the Fictitious Domain Method [16, 17]. 
A different possibility for imposing boundary conditions is the use of preexisting grid nodes. This is the case of the FM-ALE method [11, 12, 3] and the hybrid Cartesian/IBM for Cartesian grids [15, 26, 22]. In this work we will concentrate on a method of this type, where only information on the values of the unknown at mesh nodes will be used to prescribe the boundary conditions in an approximate way.

Perhaps the simplest method to impose boundary conditions on non-matching meshes that uses only degrees of freedom at mesh nodes is Nitsche's method (see for example [18]). In essence, it consists in adding a term penalizing the difference between the unknown and the boundary condition it has to satisfy, adding also some additional terms for consistency (and symmetry). In [8] we proposed a method in which mesh degrees of freedom external to the computational domain are used to impose that the boundary condition be approximated as closely as possible. Even if the method has a very good numerical performance and we favor its use in flow problems, the final system of equations is non-symmetric even for symmetric problems. The method can be related to Nitsche's method if both are viewed as methods to minimize a certain difference between the unknown and the boundary condition. See [8] for details.

In [14] a new way to prescribe Dirichlet conditions was proposed. It is based on the use of a hybrid formulation with an additional element-wise discontinuous flux field enforcing the boundary condition. This flux can be condensed, yielding a problem posed in terms of the original unknowns only. No analysis is available for this method. Its main drawback is, as for the method in [8], that the final problem is non-symmetric even if the original one is symmetric. In [4] a modification was proposed, in which new terms were added to ensure consistency and symmetry for symmetric problems. Moreover, a stability analysis was performed for the problems treated, namely, the convection-diffusion equation and the incompressible Navier-Stokes equations.

The present paper can be considered a follow up of [4]. Here we present the methodology described in this reference for an abstract elliptic problem and apply it to several examples. Apart from the Poisson problem and the Stokes problem using stabilized finite element formulations already discussed in [4], we also consider Darcy's problem, in primal and dual forms, with inf-sup stable and with stabilized formulations, as well as inf-sup stable approximations to the Stokes problem and the three-field version of it. In summary, we present nine examples, two of which already appeared in [4], and an abstract approach to the method proposed.

The new abstract approach of the method is presented in Section 2. We explain the idea starting from a classical variational description of the problem to be solved. This ensures that the final problem will be symmetric by construction. The advantage of the method proposed with respect to the related Nitsche's method is that the "penalty" parameter needs not being large for stability. In Section 3 we briefly recall the method for Poisson's problem, whereas in Section 4 we apply the formulation to Darcy's problem and in Section 5 to the Stokes problem. The results of some numerical experiments are shown in Section 6 and conclusions are drawn in Section 7.

\section{Description of the method for an abstract problem}

Let us consider an elliptic problem of the form: find $u: \Omega \longrightarrow \mathbb{R}^{n}$ such that

$$
\begin{array}{rlrl}
\mathcal{L} u & =f & & \text { in } \Omega, \\
\mathcal{D} u=\mathcal{D} \bar{u} & & \text { on } \Gamma=\partial \Omega,
\end{array}
$$

where $n$ is the number of unknowns, $\mathcal{L}$ is a symmetric linear elliptic operator, $f: \Omega \longrightarrow \mathbb{R}^{n}$ is a given force term and $\bar{u}: \Gamma \longrightarrow \mathbb{R}^{n}$ a given boundary datum. $\mathcal{D}$ is an operator on functions defined on $\Gamma$ that depends on the number of components of $u$ which can be prescribed.

Suppose that problem (1)-(2) is well posed (maybe in a distributional sense) if $u \in X_{\bar{u}}$, where $X_{\bar{u}}=\{v \in$ $X \mid \mathcal{D} v=\mathcal{D} \bar{u}\}$ and $X$ is an appropriate functional space. If $X^{\prime}$ is the dual of $X$, the duality arising from the integral will be denoted by $\langle\cdot, \cdot\rangle_{\Omega}$, whereas the duality between the corresponding space of traces $\Lambda$ and its dual $\Lambda^{\prime}$ will be denoted by $\langle\cdot, \cdot\rangle_{\Gamma}$. The $L^{2}$ inner product in a domain $\omega$ will be written as $(\cdot, \cdot)_{\omega}$.

Being $\mathcal{L}$ symmetric and elliptic, problem (1)-(2) corresponds to the Euler-Lagrange equations for the optimization of a certain functional $F: X_{\bar{u}} \longrightarrow \mathbb{R}$. For conciseness, let us assume that $F$ is positive-definite, so that this optimization is in fact a minimization, i.e.,

$$
u=\arg \inf _{v \in X_{\bar{u}}} F(v)
$$


Taking the first variation of $F$ in the direction of $\delta u \in X_{0}$, the solution to problem (3) satisfies

$$
B(u, \delta u)=L(\delta u) \quad \forall \delta u \in X_{0},
$$

where $B(u, \delta u)=\langle\mathcal{L} u, \delta u\rangle_{\Omega}, L(\delta u)=\langle f, \delta u\rangle_{\Omega}$.

Still at the continuous level, we may reformulate the previous problems (3) and (4) using Lagrange multipliers to enforce the boundary condition (2). The analogue to problem (3) consists of finding $[u, \lambda] \in X \times \Lambda^{\prime}$ as the solution to

$$
[u, \lambda]=\arg \inf _{v \in X} \sup _{\mu \in \Lambda^{\prime}}\left[F(v)-\langle\mu, \mathcal{D} v-\mathcal{D} \bar{u}\rangle_{\Gamma}\right]
$$

whereas the analogue of (4) is: find $[u, \lambda] \in X \times \Lambda^{\prime}$ such that

$$
\begin{aligned}
B(u, \delta u)-\langle\lambda, \mathcal{D} \delta u\rangle_{\Gamma} & =L(\delta u) & & \forall \delta u \in X, \\
\langle\delta \lambda, \mathcal{D} u\rangle_{\Gamma} & =\langle\delta \lambda, \mathcal{D} \bar{u}\rangle_{\Gamma} & & \forall \delta \lambda \in \Lambda^{\prime} .
\end{aligned}
$$

Note that in (6)-(7), $u, \delta u \in X$.

Let us consider now the finite element approximation to the problem. Let $\Omega_{h}$ the domain that covers $\Omega$ introduced above, and let $\mathcal{P}_{h}=\{K\}$ a finite element partition of $\Omega_{h}$ of size $h$, which for simplicity we will assume quasi-uniform throughout. All finite element spaces and functions constructed from $\mathcal{P}_{h}$ will be identified with a subscript $h$. These are assumed to be defined on $\Omega_{h}$, not only on $\Omega$. Since $\Gamma$ is interior to $\Omega_{h}$, the exact imposition of the Dirichlet condition implied by (3) and (4) cannot be translated at the discrete level. However, the discrete counterpart of (5) and (6)-(7) is straightforward. In particular, the former corresponds to the optimization of the functional

$$
G\left(\left[v_{h}, \mu_{h}\right]\right)=F\left(v_{h}\right)-\left\langle\mu_{h}, \mathcal{D} v_{h}-\mathcal{D} \bar{u}\right\rangle_{\Gamma},
$$

in $X_{h} \times \Lambda_{h}^{\prime}$, whereas the latter reads: find $\left[u_{h}, \lambda_{h}\right] \in X_{h} \times \Lambda_{h}^{\prime}$ such that

$$
\begin{aligned}
B\left(u_{h}, \delta u_{h}\right)-\left\langle\lambda_{h}, \mathcal{D} \delta u_{h}\right\rangle_{\Gamma} & =L\left(\delta u_{h}\right) & & \forall \delta u_{h} \in X_{h}, \\
\left\langle\delta \lambda_{h}, \mathcal{D} u_{h}\right\rangle_{\Gamma} & =\left\langle\delta \lambda_{h}, \mathcal{D} \bar{u}\right\rangle_{\Gamma} & & \forall \delta \lambda_{h} \in \Lambda_{h}^{\prime} .
\end{aligned}
$$

Note that the possible integrals involved in the bilinear form $B$ appearing in (6) and (9) need to be performed over $\Omega$. This may lead to the need of splitting the elements cut by $\Gamma$ for integration purposes (see [4]). Likewise, $\Gamma$ will probably need to be approximated, for example by piecewise polynomials of the same order as the finite element interpolation.

It is important to note that in order to have a well posed problem the finite element spaces $X_{h}$ and $\Lambda_{h}^{\prime}$ must satisfy an inf-sup condition, that can be expressed by saying that for each $\lambda_{h} \in \Lambda_{h}^{\prime}$ there exists $v_{h} \in X_{h} \backslash\{0\}$ such that

$$
\beta\left\|\lambda_{h}\right\|_{\Lambda^{\prime}}\left\|v_{h}\right\|_{X} \leq\left\langle\lambda_{h}, \mathcal{D} v_{h}\right\rangle_{\Gamma},
$$

for a constant $\beta>0$. Here and below, $\|\cdot\|_{Y}$ stands for the norm in a functional space $Y$.

The method we propose can be described as a modification of the previous Lagrange multiplier technique. The idea is to link the Lagrange multiplier with the variable $u_{h}$ by imposing that it is equal, in a least squares sense, to the trace on $\Gamma$ of the appropriate flux of $u_{h}$ or, when $u_{h}$ itself is a flux, by imposing that it is equal to the flux of the Lagrange multiplier. This flux depends on the problem to be solved, and some examples will be presented in the following sections. Let us write it as $\boldsymbol{\sigma}_{h}=\mathcal{F} u_{h}$, and let $\sigma_{n, h}$ be an appropriate trace of $\boldsymbol{\sigma}_{h}$ on $\Gamma$. In the case $u_{h}$ itself is a flux, we will write $u_{h}=\mathcal{F} \mu_{h}$, but let us consider the former case for the sake of conciseness. If $\Sigma_{h}$ is the space where $\sigma_{h}$ is defined, instead of the discrete version of (5) we propose to optimize the functional

$$
\hat{G}\left(\left[v_{h}, \boldsymbol{\tau}_{h}\right]\right)=F\left(v_{h}\right)-\left\langle\tau_{n, h}, \mathcal{D} v_{h}-\mathcal{D} \bar{u}\right\rangle_{\Gamma}-\frac{1}{2 N}\left\|\boldsymbol{\tau}_{h}-\mathcal{F} v_{h}\right\|_{L^{2}(\Omega)}^{2},
$$

instead of $G$ in (8), where $N$ is sufficiently large. It acts as the penalty parameter of the formulation although, as we shall see, it needs not being very large to ensure stability of the formulation. Note that at this point it is in general dimensional. In particular, if $F$ is positive defined the problem to be solved is

$$
\left[u_{h}, \boldsymbol{\sigma}_{h}\right]=\arg \inf _{v_{h} \in X_{h}} \sup _{\boldsymbol{\tau}_{h} \in \Sigma_{h}} \hat{G}\left(\left[v_{h}, \boldsymbol{\tau}_{h}\right]\right),
$$


The discrete variational equations corresponding to the optimization of (12) read as follows: find $\left[u_{h}, \boldsymbol{\sigma}_{h}\right] \in$ $X_{h} \times \Sigma_{h}$ such that

$$
\begin{array}{rlrl}
B\left(u_{h}, \delta u_{h}\right)-\left\langle\sigma_{n, h}, \mathcal{D} \delta u_{h}\right\rangle_{\Gamma}-\frac{1}{N}\left(-\mathcal{F} \delta u_{h}, \boldsymbol{\sigma}_{h}-\mathcal{F} u_{h}\right)_{\Omega} & =L\left(\delta u_{h}\right) & & \forall \delta u_{h} \in X_{h}, \\
-\left\langle\delta \sigma_{n, h}, \mathcal{D} u_{h}\right\rangle_{\Gamma}-\frac{1}{N}\left(\delta \boldsymbol{\sigma}_{h}, \boldsymbol{\sigma}_{h}-\mathcal{F} u_{h}\right)_{\Omega} & =-\left\langle\delta \sigma_{n, h}, \mathcal{D} \bar{u}\right\rangle_{\Gamma} & \forall \delta \boldsymbol{\sigma}_{h} \in \Sigma_{h} .
\end{array}
$$

This is the method that we shall apply to different problems in what follows, and whose stability will be analyzed in each case. We note that we have introduced the method at the discrete level, and in particular the norms in which stability will be proven make sense only in the discrete case.

\section{Poisson's problem}

In this section we will consider the scalar Poisson's problem, which consists of finding $u: \Omega \longrightarrow \mathbb{R}$ such that

$$
\begin{aligned}
-k \Delta u=f & \text { in } \Omega, \\
u=\bar{u} & \text { on } \Gamma,
\end{aligned}
$$

with $k>0$. Using the notation introduced in the previous section, now we have that $n=1, \mathcal{L}=-k \Delta, \mathcal{D}$ is the identity for functions defined on $\Gamma$, and:

$$
\begin{aligned}
F(v) & =\frac{1}{2} k\|\nabla v\|_{L^{2}(\Omega)}^{2}-L(v), \\
B(u, \delta u) & =k(\nabla u, \nabla \delta u)_{\Omega}, \\
\mathcal{F} u & =k \nabla u .
\end{aligned}
$$

We may also take $N=N_{0} k$, with $N_{0}$ dimensionless. The problem is well posed in $X=V=H^{1}(\Omega)$, the space of functions whose derivatives of order up to one are square integrable. The space of traces is $\Lambda=H^{1 / 2}(\Gamma)$.

Let $V_{h} \subset H^{1}\left(\Omega_{h}\right)$ be a finite element space for the discrete finite element solution $u_{h}$ and $\Sigma_{h}$ the space to approximate $\sigma_{h}$. Functional (12) is now

$$
\hat{G}\left(\left[v_{h}, \boldsymbol{\tau}_{h}\right]\right)=F\left(v_{h}\right)-\left\langle\tau_{n, h}, v_{h}-\bar{u}\right\rangle_{\Gamma}-\frac{1}{2 N_{0} k}\left\|\boldsymbol{\tau}_{h}-k \nabla v_{h}\right\|_{L^{2}(\Omega)}^{2},
$$

where in this case $\tau_{n, h}=\boldsymbol{n} \cdot \boldsymbol{\tau}_{h}$ for $\boldsymbol{\tau}_{h} \in \Sigma_{h}, \boldsymbol{n}$ being the unit normal at $\Gamma$ external to $\Omega$.

Problem (14)-(15) now reads: find $\left[u_{h}, \boldsymbol{\sigma}_{h}\right] \in V_{h} \times \Sigma_{h}$ such that

$$
\begin{array}{rlrl}
\left(1-\frac{1}{N_{0}}\right) k\left(\nabla u_{h}, \nabla \delta u_{h}\right)_{\Omega}-\left\langle\sigma_{n, h}, \delta u_{h}\right\rangle_{\Gamma}+\frac{1}{N_{0}}\left(\boldsymbol{\sigma}_{h}, \nabla \delta u_{h}\right)_{\Omega} & =L\left(\delta u_{h}\right) & & \forall \delta u_{h} \in V_{h}, \\
\left\langle\delta \sigma_{n, h}, u_{h}\right\rangle_{\Gamma}+\frac{1}{N_{0} k}\left(\delta \boldsymbol{\sigma}_{h}, \boldsymbol{\sigma}_{h}-k \nabla u_{h}\right)_{\Omega} & =\left\langle\delta \sigma_{n, h}, \bar{u}\right\rangle_{\Gamma} & \forall \delta \boldsymbol{\sigma}_{h} \in \Sigma_{h},
\end{array}
$$

Note that the problem can be easily symmetrized by changing the sign of the second equation.

Problem (19)-(20) was presented already in [4], with the only modification that in this reference $\boldsymbol{\sigma}_{h}$ is only defined on $\Omega_{\Gamma}$ (see Fig. 1), and thus the integrals involving $\sigma_{h}$ and $\delta \sigma_{h}$ need to extend only over the elements cut by $\Gamma$.

In [4] it is also explained how the method compares to the classical Nitsche's method. It is shown that in essence there is a modification of the penalty term of this method, so that instead of penalizing the difference between the unknown and the the boundary condition what is penalized is the product of this difference times a weighting term which depends on the surface to volume ratio of each boundary element. See [4] for details.

Stability of problem (19)-(20) was also proved in [4]. Here we shall repeat it with slight modifications because the ideas involved in the proof will allow us to prove stability for the other examples presented in this paper, which involve additional ingredients. 
The bilinear form associated to problem (19)-(20) is

$$
\begin{aligned}
B_{\mathrm{P}}\left(\left[u_{h}, \boldsymbol{\sigma}_{h}\right],\left[\delta u_{h}, \delta \boldsymbol{\sigma}_{h}\right]\right) & =\left(1-\frac{1}{N_{0}}\right) k\left(\nabla u_{h}, \nabla \delta u_{h}\right)_{\Omega}-\left\langle\sigma_{n, h}, \delta u_{h}\right\rangle_{\Gamma}+\frac{1}{N_{0}}\left(\boldsymbol{\sigma}_{h}, \nabla \delta u_{h}\right)_{\Omega} \\
& +\left\langle\delta \sigma_{n, h}, u_{h}\right\rangle_{\Gamma}+\frac{1}{N_{0} k}\left(\delta \boldsymbol{\sigma}_{h}, \boldsymbol{\sigma}_{h}-k \nabla u_{h}\right)_{\Omega} .
\end{aligned}
$$

We shall prove that this bilinear form is stable in the norm

$$
\left\|\left[u_{h}, \boldsymbol{\sigma}_{h}\right]\right\|_{\mathrm{P}}^{2}:=k\left\|\nabla u_{h}\right\|_{L^{2}(\Omega)}^{2}+\frac{k}{h}\left\|u_{h}\right\|_{L^{2}(\Gamma)}^{2}+\frac{1}{k}\|\boldsymbol{\sigma}\|_{L^{2}(\Omega)}^{2} .
$$

Recall that $h$ is the size of the finite element partition, which we assume quasi-uniform for the sake of simplicity.

Stability of (21) relies on the following a compatibility condition between the spaces $V_{h}$ and $\Sigma_{h}$ :

$$
\begin{aligned}
& \forall v_{h} \in V_{h} \quad \exists \boldsymbol{\tau}_{h} \in \Sigma_{h}, \delta_{0}>0 \text { such that }\left\|v_{h}\right\|_{L^{2}(\Gamma)}^{2} \lesssim\left\langle\boldsymbol{\tau}_{h} \cdot \boldsymbol{n}, v_{h}\right\rangle_{\Gamma}+\delta_{0} h\left\|\nabla v_{h}\right\|_{L^{2}(\Omega)}^{2}, \\
& \left\|\boldsymbol{\tau}_{h}\right\|_{L^{2}(\Gamma)}=\left\|v_{h}\right\|_{L^{2}(\Gamma)}, \quad\left\|\boldsymbol{\tau}_{h}\right\|_{L^{2}(\Omega)}^{2} \lesssim h\left\|v_{h}\right\|_{L^{2}(\Gamma)}^{2} .
\end{aligned}
$$

Here and below we use the symbol $\lesssim$ for $\leq$ up to positive dimensionless constants, and likewise for $\gtrsim$.

It is shown in [4] that conditions (23)-(24) hold in two cases of practical interest, namely, the case in which continuous equal interpolation is used for both $V_{h}$ and $\Sigma_{h}$ and the case in which $V_{h}$ is made of continuous linear interpolations and $\Sigma_{h}$ of discontinuous piecewise constants. The latter is particularly interesting, since it allows one to eliminate the degrees of freedom associated to $\sigma_{h}$ in (19)-(20) and end up with a problem posed in terms of the degrees of freedom of $u_{h}$ only.

The stability result for (21) is the following:

Theorem 1. Assume that (23)-(24) hold and that $N_{0}>1$ in (21). Then, for all $\left[u_{h}, \sigma_{h}\right]$ there exists a non trivial $\left[\delta u_{h}, \delta \boldsymbol{\sigma}_{h}\right]$ such that:

$$
B_{\mathrm{P}}\left(\left[u_{h}, \boldsymbol{\sigma}_{h}\right],\left[\delta u_{h}, \delta \boldsymbol{\sigma}_{h}\right]\right) \gtrsim\left\|\left[u_{h}, \boldsymbol{\sigma}_{h}\right]\right\|_{\mathrm{P}}\left\|\left[\delta u_{h}, \delta \boldsymbol{\sigma}_{h}\right]\right\|_{\mathrm{P}} .
$$

Proof. Let us start noting that for $N_{0}>1$

$$
B_{\mathrm{P}}\left(\left[u_{h}, \boldsymbol{\sigma}_{h}\right],\left[u_{h}, \boldsymbol{\sigma}_{h}\right]\right) \gtrsim k\left\|\nabla u_{h}\right\|_{L^{2}(\Omega)}^{2}+\frac{1}{k}\|\boldsymbol{\sigma}\|_{L^{2}(\Omega)}^{2} .
$$

Given $u_{h}$, let $\tau_{h}^{u}$ be the element in $\Sigma_{h}$ that satisfies (23)-(24) with $v_{h}=u_{h}$. We have that

$$
\begin{aligned}
B_{\mathrm{P}}\left(\left[u_{h}, \boldsymbol{\sigma}_{h}\right],\left[0, h^{-1} k \boldsymbol{\tau}_{h}^{u}\right]\right) & \gtrsim \frac{k}{h}\left\|u_{h}\right\|_{L^{2}(\Gamma)}^{2}-\delta_{0} k\left\|\nabla u_{h}\right\|_{L^{2}(\Omega)}^{2} \\
& -\frac{1}{N_{0} h^{1 / 2}}\left\|u_{h}\right\|_{L^{2}(\Gamma)}\left(\left\|\boldsymbol{\sigma}_{h}\right\|_{L^{2}(\Omega)}+k\left\|\nabla u_{h}\right\|_{L^{2}(\Omega)}\right) .
\end{aligned}
$$

After using Young's inequality in the last terms we arrive at

$$
B_{\mathrm{P}}\left(\left[u_{h}, \boldsymbol{\sigma}_{h}\right],\left[0, h^{-1} k \boldsymbol{\tau}_{h}^{u}\right]\right) \gtrsim \frac{k}{h}\left\|u_{h}\right\|_{L^{2}(\Gamma)}^{2}-k\left\|\nabla u_{h}\right\|_{L^{2}(\Omega)}^{2}-\frac{1}{k}\left\|\boldsymbol{\sigma}_{h}\right\|_{L^{2}(\Omega)}^{2} .
$$

From (25)-(26) it follows that

$$
B_{\mathrm{P}}\left(\left[u_{h}, \boldsymbol{\sigma}_{h}\right],\left[u_{h}, \boldsymbol{\sigma}_{h}+\beta h^{-1} k \boldsymbol{\tau}_{h}^{u}\right]\right) \gtrsim\left\|\left[u_{h}, \boldsymbol{\sigma}_{h}\right]\right\|_{\mathrm{P}}^{2}
$$

if $\beta$ is small enough. It is easily seen that if $\left[\delta u_{h}, \delta \boldsymbol{\sigma}_{h}\right]=\left[u_{h}, \boldsymbol{\sigma}_{h}+\beta h^{-1} k \boldsymbol{\tau}_{h}^{u}\right]$, then $\left\|\left[u_{h}, \boldsymbol{\sigma}_{h}\right]\right\|_{\mathrm{P}} \gtrsim$ $\left\|\left[\delta u_{h}, \delta \boldsymbol{\sigma}_{h}\right]\right\|_{\mathrm{P}}$, from where the theorem follows.

Once stability is established, optimal convergence can be proven using more or less conventional techniques, since the method can be understood as a consistent method introducing the appropriate unknowns. The convergence analysis is however beyond the scope of this paper. 


\section{Darcy's problem}

In this section we shall apply the general methodology described in Section 2 to the mixed form of the Poisson equation, which consists of finding $u: \Omega \longrightarrow \mathbb{R}$ and $\boldsymbol{q}: \Omega \longrightarrow \mathbb{R}^{d}$ such that

$$
\begin{aligned}
& \frac{1}{k} \boldsymbol{q}+\nabla u=\mathbf{0} \quad \text { in } \Omega, \\
& \nabla \cdot \boldsymbol{q}=f \quad \text { in } \Omega,
\end{aligned}
$$

and satisfying appropriate essential boundary conditions. These depend on the functional setting of the problem, leading to the so called mixed primal and mixed dual forms of Darcy's problem (see [5]). Moreover, the finite element approximation requires either to satisfy inf-sup conditions between the approximating spaces for $u$ and $\boldsymbol{q}$ or to use stabilization techniques (see [1]). Thus, in this section we shall in fact describe the approximation to Darcy's problem with weak imposition of boundary conditions and prove its stability in four cases.

\subsection{Primal form of Darcy's problem}

In the primal form of Darcy's problem equations (27)-(28) are understood (in a distributional sense) with $u \in$ $H^{1}(\Omega)$ and $\boldsymbol{q} \in L^{2}(\Omega)^{d}$. Thus, only traces of $u$ exist on $\Gamma$, and therefore essential boundary conditions consist of imposing $u$ there. The problem to be solved is thus that of finding $u$ and $\boldsymbol{q}$ satisfying

$$
\begin{aligned}
& \frac{1}{k} \boldsymbol{q}+\nabla u=\mathbf{0} \quad \text { in } \Omega \text {, } \\
& \nabla \cdot \boldsymbol{q}=f \quad \text { in } \Omega, \\
& u=\bar{u} \quad \text { on } \Gamma \text {. }
\end{aligned}
$$

In this case the problem is exactly equivalent to (16)-(17), so that at the continuous level it has no interest. However, at the discrete level the fact that variables $u$ and $\boldsymbol{q}$ may be interpolated arbitrarily opens the door to approximations different to that of the classical Poisson's problem.

Using the notation introduced in Section 2 and assuming that the unknown is organized in the form $[\boldsymbol{q}, u]$, we now have that $n=d+1, \mathcal{L}([\boldsymbol{q}, u])=\left[\frac{1}{k} \boldsymbol{q}+\nabla u, \nabla \cdot \boldsymbol{q}\right], \mathcal{D}([\boldsymbol{q}, u])=u$ and

$$
\begin{aligned}
F([\boldsymbol{r}, v]) & =-\frac{1}{2 k}\|\boldsymbol{r}\|_{L^{2}(\Omega)}^{2}-(\nabla v, \boldsymbol{r})_{\Omega}-\langle f, v\rangle_{\Omega}, \\
B([\boldsymbol{q}, u],[\delta \boldsymbol{q}, \delta u]) & =-\frac{1}{k}(\boldsymbol{q}, \delta \boldsymbol{q})_{\Omega}-(\nabla u, \delta \boldsymbol{q})_{\Omega}-(\nabla \delta u, \boldsymbol{q})_{\Omega}, \\
L([\delta \boldsymbol{q}, \delta u]) & =\langle f, \delta u\rangle_{\Omega}, \\
\mathcal{F}([\boldsymbol{q}, u]) & =-\boldsymbol{q} .
\end{aligned}
$$

As for Poisson's problem, we may take $N=N_{0} k$, with $N_{0}$ dimensionless. The problem is well posed in $X=$ $L^{2}(\Omega)^{d} \times H^{1}(\Omega)$, the space of traces being $\Lambda=H^{1 / 2}(\Gamma)$ (the trace operator from $X$ to $\Lambda$ is $\left.[\boldsymbol{r}, v] \mapsto v\right|_{\Gamma}$ ). Note that now (29)-(31) are the equations corresponding to a saddle point of $F$, and not to a minimum.

\subsubsection{Galerkin finite element approximation}

Let $V_{h} \subset H^{1}\left(\Omega_{h}\right)$ be the finite element space to approximate $u, R_{h} \subset L^{2}\left(\Omega_{h}\right)^{d}$ the finite element space to approximate $\boldsymbol{q}$ and $\Sigma_{h}$ the space to approximate the flux to prescribe in a weak form (31). Following the ideas of Section 2, the method we propose consists in optimizing the functional

$$
\hat{G}\left(\left[\boldsymbol{r}_{h}, v_{h}, \boldsymbol{\tau}_{h}\right]\right)=F\left(\left[\boldsymbol{r}_{h}, v_{h}\right]\right)-\left\langle\tau_{n, h}, u_{h}-\bar{u}\right\rangle_{\Gamma}-\frac{1}{2 N_{0} k}\left\|\boldsymbol{\tau}_{h}+\boldsymbol{r}_{h}\right\|_{L^{2}(\Omega)}^{2},
$$

over $R_{h} \times V_{h} \times \Sigma_{h}$, which corresponds to (12) for the problem we are considering. As for Poisson's problem, $\tau_{n, h}=\boldsymbol{n} \cdot \boldsymbol{\tau}_{h}$. 
Problem (14)-(15) now reads: find $\left[\boldsymbol{q}_{h}, u_{h}, \boldsymbol{\sigma}_{h}\right] \in R_{h} \times V_{h} \times \Sigma_{h}$ such that

$$
\begin{aligned}
-\frac{1}{k}\left(\boldsymbol{q}_{h}, \delta \boldsymbol{q}_{h}\right)_{\Omega}-\left(\nabla u_{h}, \delta \boldsymbol{q}_{h}\right)_{\Omega}-\frac{1}{N_{0} k}\left(\delta \boldsymbol{q}_{h}, \boldsymbol{\sigma}_{h}+\boldsymbol{q}_{h}\right)_{\Omega} & =0 & & \forall \delta \boldsymbol{q}_{h} \in R_{h}, \\
-\left(\nabla \delta u_{h}, \boldsymbol{q}_{h}\right)_{\Omega}-\left\langle\sigma_{n, h}, \delta u_{h}\right\rangle_{\Gamma} & =\left\langle f, \delta u_{h}\right\rangle_{\Omega} & & \forall \delta u_{h} \in V_{h}, \\
-\left\langle\delta \sigma_{n, h}, u_{h}\right\rangle_{\Gamma}-\frac{1}{N_{0} k}\left(\delta \boldsymbol{\sigma}_{h}, \boldsymbol{\sigma}_{h}+\boldsymbol{q}_{h}\right)_{\Omega} & =-\left\langle\delta \sigma_{n, h}, \bar{u}\right\rangle_{\Gamma} & & \forall \delta \boldsymbol{\sigma}_{h} \in \Sigma_{h} .
\end{aligned}
$$

This is the formulation we propose to approximate in a weak sense boundary condition (31) for Darcy's problem in primal form and using the Galerkin finite element approximation. Note that the solution is $\boldsymbol{\sigma}_{h} \approx \boldsymbol{q}_{h}$, but in a weak sense. Variable $\sigma_{h}$ allows one to prescribe (31) and its interpolation can be completely different to that of $\boldsymbol{q}_{h}$. In particular, as for Poisson's problem, it can be condensed at the element level if it is discontinuous.

It has to be remarked that the term $\left\langle\sigma_{n, h}, \delta u_{h}\right\rangle_{\Gamma}$ in (33) would not be well defined at the continuous level if $\sigma_{n, h}$ is replaced by $\boldsymbol{n} \cdot \boldsymbol{q}$, since this term is not defined on $\Gamma$. Thus, the equations written make only sense at the discrete level and introducing the new variable $\boldsymbol{\sigma}_{h}$.

In the following we shall prove that this formulation is stable. In order to guarantee this, we will require two conditions. The first is a relationship between $V_{h}$ and $\Sigma_{h}$ that is exactly the same as for Poisson's problem, that is to say, we assume that (23)-(24) hold. The second is quite natural: we also assume that the classical infsup condition between $R_{h}$ and $V_{h}$ required for the well-posedness of the problem when Dirichlet conditions are prescribed holds, that is to say,

$$
\forall v_{h} \in V_{h} \quad \exists \boldsymbol{r}_{h} \in R_{h} \backslash\{\mathbf{0}\} \text { such that } \beta\left\|\nabla v_{h}\right\|_{L^{2}(\Omega)}\left\|\boldsymbol{r}_{h}\right\|_{L^{2}(\Omega)} \leq\left(\nabla v_{h}, \boldsymbol{r}_{h}\right)_{\Omega},
$$

for a constant $\beta>0$. Note that the continuous counterpart of (35) is automatic, since for $v \in H^{1}(\Omega)$ it suffices to take $\boldsymbol{r}=\nabla v$ to check that it holds with $\beta=1$. This is also true whenever functions in $R_{h}$ are gradients of functions in $V_{h}$, but not for arbitrary interpolations. Note that we assume in this case that (35) holds with the integrals performed over $\Omega$. In other cases this would be too restrictive, and we will need to assume classical inf-sup conditions with integrals taken over $\Omega_{h}$.

The bilinear form associated to problem (32)-(34) is given by

$$
\begin{aligned}
B_{\mathrm{DPG}}\left(\left[\boldsymbol{q}_{h}, u_{h}, \boldsymbol{\sigma}_{h}\right],\left[\delta \boldsymbol{q}_{h}, \delta u_{h}, \delta \boldsymbol{\sigma}_{h}\right]\right) & =-\frac{1}{k}\left(\boldsymbol{q}_{h}, \delta \boldsymbol{q}_{h}\right)_{\Omega}-\left(\nabla u_{h}, \delta \boldsymbol{q}_{h}\right)_{\Omega}-\left(\nabla \delta u_{h}, \boldsymbol{q}_{h}\right)_{\Omega} \\
& -\left\langle\sigma_{n, h}, \delta u_{h}\right\rangle_{\Gamma}-\left\langle\delta \sigma_{n, h}, u_{h}\right\rangle_{\Gamma}-\frac{1}{N_{0} k}\left(\delta \boldsymbol{\sigma}_{h}+\delta \boldsymbol{q}_{h}, \boldsymbol{\sigma}_{h}+\boldsymbol{q}_{h}\right)_{\Omega} .
\end{aligned}
$$

We next prove that it is stable in the norm

$$
\left\|\left[\boldsymbol{q}_{h}, u_{h}, \boldsymbol{\sigma}_{h}\right]\right\|_{\mathrm{DPG}}^{2}:=\frac{1}{k}\left\|\boldsymbol{q}_{h}\right\|_{L^{2}(\Omega)}^{2}+k\left\|\nabla u_{h}\right\|_{L^{2}(\Omega)}^{2}+\frac{k}{h}\left\|u_{h}\right\|_{L^{2}(\Gamma)}^{2}+\frac{1}{k}\left\|\boldsymbol{\sigma}_{h}\right\|_{L^{2}(\Omega)}^{2} .
$$

Theorem 2. Assume that (23)-(24) hold, that $N_{0}>1$ and that (35) also holds. Then, for all $\left[\boldsymbol{q}_{h}, u_{h}, \boldsymbol{\sigma}_{h}\right]$ there exists a non trivial $\left[\delta \boldsymbol{q}_{h}, \delta u_{h}, \delta \boldsymbol{\sigma}_{h}\right]$ such that:

$$
B_{\mathrm{DPG}}\left(\left[\boldsymbol{q}_{h}, u_{h}, \boldsymbol{\sigma}_{h}\right],\left[\delta \boldsymbol{q}_{h}, \delta u_{h}, \delta \boldsymbol{\sigma}_{h}\right]\right) \gtrsim\left\|\left[\boldsymbol{q}_{h}, u_{h}, \boldsymbol{\sigma}_{h}\right]\right\|_{\mathrm{DPG}}\left\|\left[\delta \boldsymbol{q}_{h}, \delta u_{h}, \delta \boldsymbol{\sigma}_{h}\right]\right\|_{\mathrm{DPG}} \cdot
$$

Proof. Note first that for $N_{0}>1$ we have

$$
\begin{aligned}
B_{\mathrm{DPG}}\left(\left[\boldsymbol{q}_{h}, u_{h}, \boldsymbol{\sigma}_{h}\right],\left[-\boldsymbol{q}_{h}, u_{h},-\boldsymbol{\sigma}_{h}\right]\right) & =\frac{1}{k}\left\|\boldsymbol{q}_{h}\right\|_{L^{2}(\Omega)}^{2}+\frac{1}{N_{0} k}\left\|\boldsymbol{\sigma}_{h}+\boldsymbol{q}_{h}\right\|_{L^{2}(\Omega)}^{2} \\
& \gtrsim \frac{1}{k}\left\|\boldsymbol{q}_{h}\right\|_{L^{2}(\Omega)}^{2}+\frac{1}{k}\left\|\boldsymbol{\sigma}_{h}\right\|_{L^{2}(\Omega)}^{2} .
\end{aligned}
$$

Let $\boldsymbol{r}_{h}^{u}$ the function that allows one to guarantee (35) for $v_{h}=u_{h}$. We may normalize it so that $\left\|\boldsymbol{r}_{h}^{u}\right\|_{L^{2}(\Omega)}=$ $\left\|\nabla u_{h}\right\|_{L^{2}(\Omega)}$ and we have that

$$
\begin{aligned}
B_{\mathrm{DPG}}\left(\left[\boldsymbol{q}_{h}, u_{h}, \boldsymbol{\sigma}_{h}\right],\left[-k \boldsymbol{r}_{h}^{u}, 0, \mathbf{0}\right]\right) & \gtrsim k\left\|\nabla u_{h}\right\|_{L^{2}(\Omega)}^{2}-\left\|\nabla u_{h}\right\|_{L^{2}(\Omega)}\left\|\boldsymbol{q}_{h}\right\|_{L^{2}(\Omega)} \\
& -\frac{1}{N_{0}}\left\|\nabla u_{h}\right\|_{L^{2}(\Omega)}\left(\left\|\boldsymbol{q}_{h}\right\|_{L^{2}(\Omega)}+\left\|\boldsymbol{\sigma}_{h}\right\|_{L^{2}(\Omega)}\right) .
\end{aligned}
$$


Using Young's inequality for the last terms we obtain

$$
B_{\mathrm{DPG}}\left(\left[\boldsymbol{q}_{h}, u_{h}, \boldsymbol{\sigma}_{h}\right],\left[-k \boldsymbol{r}_{h}^{u}, 0, \mathbf{0}\right]\right) \gtrsim k\left\|\nabla u_{h}\right\|_{L^{2}(\Omega)}^{2}-\frac{1}{k}\left\|\boldsymbol{q}_{h}\right\|_{L^{2}(\Omega)}^{2}-\frac{1}{N_{0} k}\left\|\boldsymbol{\sigma}_{h}\right\|_{L^{2}(\Omega)}^{2} .
$$

Given $u_{h}$, let now $\tau_{h}^{u}$ be the element in $\Sigma_{h}$ that satisfies (23)-(24) with $v_{h}=u_{h}$. We have that

$$
\begin{aligned}
B_{\mathrm{DPG}}\left(\left[\boldsymbol{q}_{h}, u_{h}, \boldsymbol{\sigma}_{h}\right],\left[0, \mathbf{0},-h^{-1} k \boldsymbol{\tau}_{h}^{u}\right]\right) & \gtrsim \frac{k}{h}\left\|u_{h}\right\|_{L^{2}(\Gamma)}^{2}-\delta_{0} k\left\|\nabla u_{h}\right\|_{L^{2}(\Omega)}^{2} \\
& -\frac{1}{N_{0} h^{1 / 2}}\left\|u_{h}\right\|_{L^{2}(\Gamma)}\left(\left\|\boldsymbol{\sigma}_{h}\right\|_{L^{2}(\Omega)}+\left\|\boldsymbol{q}_{h}\right\|_{L^{2}(\Omega)}\right) .
\end{aligned}
$$

After using Young's inequality in the last terms we arrive at

$$
\begin{aligned}
B_{\mathrm{DPG}}\left(\left[\boldsymbol{q}_{h}, u_{h}, \boldsymbol{\sigma}_{h}\right],\left[\mathbf{0}, 0,-h^{-1} k \boldsymbol{\tau}_{h}^{u}\right]\right) & \gtrsim \frac{k}{h}\left\|u_{h}\right\|_{L^{2}(\Gamma)}^{2}-k\left\|\nabla u_{h}\right\|_{L^{2}(\Omega)}^{2} \\
& -\frac{1}{k}\left\|\boldsymbol{\sigma}_{h}\right\|_{L^{2}(\Omega)}^{2}-\frac{1}{k}\left\|\boldsymbol{q}_{h}\right\|_{L^{2}(\Omega)}^{2} .
\end{aligned}
$$

From (38), (39) and (40) it follows that

$$
B_{\mathrm{DPG}}\left(\left[\boldsymbol{q}_{h}, u_{h}, \boldsymbol{\sigma}_{h}\right],\left[-\boldsymbol{q}_{h}-\beta_{1} k \boldsymbol{r}_{h}^{u}, u_{h},-\boldsymbol{\sigma}_{h}-\beta_{2} h^{-1} k \boldsymbol{\tau}_{h}^{u}\right]\right) \gtrsim\left\|\left[\boldsymbol{q}_{h}, u_{h}, \boldsymbol{\sigma}_{h}\right]\right\|_{\mathrm{DPG}}^{2}
$$

if $\beta_{1}$ and $\beta_{2}$ are small enough. It is easily seen that if $\left[\delta \boldsymbol{q}_{h}, \delta u_{h}, \delta \boldsymbol{\sigma}_{h}\right]=\left[-\boldsymbol{q}_{h}-\beta_{1} k \boldsymbol{r}_{h}^{u}, u_{h},-\boldsymbol{\sigma}_{h}-\beta_{2} h^{-1} k \boldsymbol{\tau}_{h}^{u}\right]$, then $\left\|\left[\boldsymbol{q}_{h}, u_{h}, \boldsymbol{\sigma}_{h}\right]\right\|_{\mathrm{DPG}} \gtrsim\left\|\left[\delta \boldsymbol{q}_{h}, \delta u_{h}, \delta \boldsymbol{\sigma}_{h}\right]\right\|_{\mathrm{DPG}}$, and the proof is complete.

\subsubsection{Stabilized finite element approximation}

The Galerkin finite element approximation described above requires the compatibility condition (35) for being stable. Except for cases in which $R_{h}$ is made of functions of the form $\nabla v_{h}$, with $v_{h} \in V_{h}$, it is not easy to satisfy. In order to avoid the need to satisfy it, it is possible to use stabilized finite element methods. It is not our purpose here neither to motivate nor to discuss them in detail. We will simply use the simplest stabilization method presented in [1, 2] for the Darcy and the Stokes problems. In fact, we favor the orthogonal subscales stabilization (OSS) introduced in [6], but we will use the most popular stabilization method because our only objective is to show how stabilization and the weak imposition of essential boundary conditions presented in this paper work together.

The stabilized finite element method we consider reads as follows: find $\left[\boldsymbol{q}_{h}, u_{h}, \boldsymbol{\sigma}_{h}\right] \in R_{h} \times V_{h} \times \Sigma_{h}$ such that

$$
B_{\mathrm{DPS}}\left(\left[\boldsymbol{q}_{h}, u_{h}, \boldsymbol{\sigma}_{h}\right],\left[\delta \boldsymbol{q}_{h}, \delta u_{h}, \delta \boldsymbol{\sigma}_{h}\right]\right)=L_{\mathrm{DPS}}\left(\left[\delta \boldsymbol{q}_{h}, \delta u_{h}, \delta \boldsymbol{\sigma}_{h}\right]\right),
$$

for all $\left[\delta \boldsymbol{q}_{h}, \delta u_{h}, \delta \boldsymbol{\sigma}_{h}\right] \in R_{h} \times V_{h} \times \Sigma_{h}$, where

$$
\begin{aligned}
B_{\mathrm{DPS}}\left(\left[\boldsymbol{q}_{h}, u_{h}, \boldsymbol{\sigma}_{h}\right],\left[\delta \boldsymbol{q}_{h}, \delta u_{h}, \delta \boldsymbol{\sigma}_{h}\right]\right) & =B_{\mathrm{DPG}}\left(\left[\boldsymbol{q}_{h}, u_{h}, \boldsymbol{\sigma}_{h}\right],\left[\delta \boldsymbol{q}_{h}, \delta u_{h}, \delta \boldsymbol{\sigma}_{h}\right]\right) \\
& +\sum_{K} \tau_{q}\left(\frac{1}{k} \delta \boldsymbol{q}_{h}+\nabla \delta u_{h}, \frac{1}{k} \boldsymbol{q}_{h}+\nabla u_{h}\right)_{K \cap \Omega} \\
& -\sum_{K} \tau_{u}\left(\nabla \cdot \boldsymbol{q}_{h}, \nabla \cdot \delta \boldsymbol{q}_{h}\right)_{K \cap \Omega} \\
L_{\mathrm{DPS}}\left(\left[\delta \boldsymbol{q}_{h}, \delta u_{h}, \delta \boldsymbol{\sigma}_{h}\right]\right) & =\left\langle f, \delta u_{h}\right\rangle_{\Omega}-\left\langle\delta \sigma_{n, h}, \bar{u}\right\rangle_{\Gamma}-\sum_{K} \tau_{u}\left(\nabla \cdot \delta \boldsymbol{q}_{h}, f\right)_{K \cap \Omega},
\end{aligned}
$$

with $B_{\mathrm{DPG}}$ given by (36) and where $\tau_{q}$ and $\tau_{u}$ are the so called stabilization parameters. In order to get optimal stability and convergence for the primal form of Darcy's problem they need to be computed as

$$
\tau_{q}=k \tau_{q}^{0}, \quad \tau_{u}=\frac{h^{2}}{k} \tau_{u}^{0}
$$




\begin{tabular}{|c|c|c|c|}
\hline $\boldsymbol{K}_{q q} \boldsymbol{Q}$ & $\boldsymbol{K}_{q u} \boldsymbol{U}$ & $\boldsymbol{K}_{q \sigma} \boldsymbol{\Sigma}$ & $\boldsymbol{K}_{u q} \boldsymbol{Q}$ \\
\hline$\frac{1}{k}\left(\boldsymbol{q}_{h}, \delta \boldsymbol{q}_{h}\right)_{\Omega}$ & $\left(\nabla u_{h}, \delta \boldsymbol{q}_{h}\right)_{\Omega}$ & $\frac{1}{k}\left(\delta \boldsymbol{q}_{h}, \boldsymbol{\sigma}_{h}\right)_{\Omega}$ & $\overline{\left(\bar{c}\left(\nabla \delta u_{h}, \boldsymbol{q}_{h}\right)_{\Omega}\right.}$ \\
\hline$G_{u \sigma} \Sigma$ & $\overline{F_{u}}$ & $\boldsymbol{G}_{\sigma u} \boldsymbol{U}$ & $\boldsymbol{K}_{\sigma q} \boldsymbol{Q}$ \\
\hline \multirow[t]{3}{*}{$<\sigma_{n, h}, \delta u_{h}>$} & $>_{\Gamma} \mid\left\langle f, \delta u_{h}\right\rangle_{\Omega}$ & $\left\langle\delta \boldsymbol{\sigma}_{h}, u_{h}\right\rangle_{\Gamma}$ & $\frac{1}{k}\left(\delta \boldsymbol{\sigma}_{h}, \boldsymbol{q}_{h}\right)_{\Omega}$ \\
\hline & $\overline{G_{q u} U}$ & $\overline{\boldsymbol{G}_{u q} \boldsymbol{U}}$ & \\
\hline & $\left.{ }_{h}, u_{h}\right\rangle_{\Gamma}$ & $\left\langle\boldsymbol{n} \cdot \boldsymbol{q}_{h}, \delta u_{r}\right.$ & \\
\hline
\end{tabular}

Table 1: Algebraic terms for the primal form of Darcy's problem.

with $\tau_{q}^{0}$ and $\tau_{u}^{0}$ dimensionless algorithmic constants (see [1,2] for details). The need for having $\tau_{q}^{0}>0, \tau_{u}^{0}>0$ (in general) is the alternative to satisfy condition (35).

We next show that the bilinear form $B_{\mathrm{DPS}}$ is stable in the norm

$$
\left\|\left[\boldsymbol{q}_{h}, u_{h}, \boldsymbol{\sigma}_{h}\right]\right\|_{\mathrm{DPS}}^{2}:=\left\|\left[\boldsymbol{q}_{h}, u_{h}, \boldsymbol{\sigma}_{h}\right]\right\|_{\mathrm{DPG}}^{2}+\tau_{u}^{0} \frac{h^{2}}{k}\left\|\nabla \cdot \boldsymbol{q}_{h}\right\|_{L^{2}(\Omega)}^{2} .
$$

where $\left\|\left[\boldsymbol{q}_{h}, u_{h}, \boldsymbol{\sigma}_{h}\right]\right\|_{\mathrm{DPG}}^{2}$ is defined in (37). The last term in fact provides no significant control on the divergence of $\boldsymbol{q}_{h}$, but appears if $\tau_{u}^{0}>0$. In this case we may safely take $\tau_{u}^{0}=0$.

Theorem 3. Assume that (23)-(24) hold, that $N_{0}>1$ and that $0<\tau_{q}^{0}<1$ in (44). Then, for all $\left[\boldsymbol{q}_{h}, u_{h}, \boldsymbol{\sigma}_{h}\right]$ there exists a non trivial $\left[\delta \boldsymbol{q}_{h}, \delta u_{h}, \delta \boldsymbol{\sigma}_{h}\right]$ such that:

$$
B_{\mathrm{DPS}}\left(\left[\boldsymbol{q}_{h}, u_{h}, \boldsymbol{\sigma}_{h}\right],\left[\delta \boldsymbol{q}_{h}, \delta u_{h}, \delta \boldsymbol{\sigma}_{h}\right]\right) \gtrsim\left\|\left[\boldsymbol{q}_{h}, u_{h}, \boldsymbol{\sigma}_{h}\right]\right\|_{\mathrm{DPS}}\left\|\left[\delta \boldsymbol{q}_{h}, \delta u_{h}, \delta \boldsymbol{\sigma}_{h}\right]\right\|_{\mathrm{DPS}} .
$$

Proof. The proof is similar to that of Theorem 3, and thus we will only stress the difference. For $N_{0}>1$ and $\tau_{q}^{0}<1$ we immediately get

$$
\begin{aligned}
B_{\mathrm{DPS}}\left(\left[\boldsymbol{q}_{h}, u_{h}, \boldsymbol{\sigma}_{h}\right],\left[-\boldsymbol{q}_{h}, u_{h},-\boldsymbol{\sigma}_{h}\right]\right) & \gtrsim \frac{1}{k}\left\|\boldsymbol{q}_{h}\right\|_{L^{2}(\Omega)}^{2}+\frac{1}{k}\left\|\boldsymbol{\sigma}_{h}\right\|_{L^{2}(\Omega)}^{2} \\
& +k\left\|\nabla u_{h}\right\|_{L^{2}(\Omega)}^{2}+\tau_{u}^{0} \frac{h^{2}}{k}\left\|\nabla \cdot \boldsymbol{q}_{h}\right\|_{L^{2}(\Omega)}^{2},
\end{aligned}
$$

so that now we do not need (35) to get control on the gradient of $u_{h}$. It only remains to get control on the boundary norm of $u_{h}$, which can be done exactly as in Theorem 2 (note that the stabilization terms do not involve the extra unknown $\sigma_{h}$ ). Details are omitted.

\subsubsection{Matrix structure}

Let us now study the matrix structure of the final problem. We will focus in the Galerkin form of the problem, and we will consider the space for the Lagrange multipliers to be rich enough and elementwise discontinuous so that they can be condensed. After operating, We will arrive to a method which is very similar to Nitsche's method, the only difference being in the definition of the penalty term.

The associated algebraic form of the Galerkin primal form of Darcy problem is:

$$
\begin{aligned}
-\boldsymbol{K}_{q q} \boldsymbol{Q}-\boldsymbol{K}_{q u} \boldsymbol{U}-\frac{1}{N_{0}} \boldsymbol{K}_{q q} \boldsymbol{Q}-\frac{1}{N_{0}} \boldsymbol{K}_{q \sigma} \boldsymbol{\Sigma} & =0, \\
-\boldsymbol{K}_{u q} \boldsymbol{Q}-\boldsymbol{G}_{u \sigma} \boldsymbol{\Sigma} & =\boldsymbol{F}_{u}, \\
-\boldsymbol{G}_{\sigma u} \boldsymbol{U}-\frac{1}{N_{0}} \boldsymbol{K}_{\sigma \sigma} \boldsymbol{\Sigma}-\frac{1}{N_{0}} \boldsymbol{K}_{\sigma q} \boldsymbol{Q} & =-\boldsymbol{G}_{\sigma u} \overline{\boldsymbol{U}}
\end{aligned}
$$

the description of each term is presented in Table 1.

Let us suppose that $\Sigma_{h}$ is chosen such that $R_{h} \subset \Sigma_{h}$, and $\Sigma_{h}$ is elementwise discontinuous, then: 


$$
\forall \boldsymbol{q}_{h} \in R_{h}, \quad P_{\Sigma_{h}}\left(\boldsymbol{q}_{h}\right)=\boldsymbol{q}_{h}
$$

From (48):

$$
\boldsymbol{\Sigma}=N_{0} \boldsymbol{K}_{\sigma \sigma}^{-1}\left(\boldsymbol{G}_{\sigma u} \boldsymbol{U}-\boldsymbol{G}_{\sigma u} \overline{\boldsymbol{U}}\right)-\boldsymbol{K}_{\sigma \sigma}^{-1} \boldsymbol{K}_{\sigma q} \boldsymbol{Q} .
$$

Replacing in (46):

$$
-\boldsymbol{K}_{q q} \boldsymbol{Q}-\boldsymbol{K}_{q u} \boldsymbol{U}-\frac{1}{N_{0}} \boldsymbol{K}_{q q} \boldsymbol{Q}-\frac{1}{N_{0}} \boldsymbol{K}_{q \sigma}\left[N_{0} \boldsymbol{K}_{\sigma \sigma}^{-1}\left(\boldsymbol{G}_{\sigma u} \boldsymbol{U}-\boldsymbol{G}_{\sigma u} \overline{\boldsymbol{U}}\right)-\boldsymbol{K}_{\sigma \sigma}^{-1} \boldsymbol{K}_{\sigma q} \boldsymbol{Q}\right]=0 .
$$

Since $R_{h} \subset \Sigma_{h}$,

$$
\boldsymbol{K}_{q \sigma} \boldsymbol{K}_{\sigma \sigma}^{-1} \boldsymbol{K}_{\sigma q} \boldsymbol{Q}=\boldsymbol{K}_{q q} \boldsymbol{Q}
$$

Similarly:

$$
\boldsymbol{K}_{q \sigma} \boldsymbol{K}_{\sigma \sigma}^{-1} \boldsymbol{G}_{\sigma u} \boldsymbol{U}=\boldsymbol{G}_{q u} \boldsymbol{U}
$$

so (46) results in:

$$
-\boldsymbol{K}_{q q} \boldsymbol{Q}-\boldsymbol{K}_{q u} \boldsymbol{U}-\boldsymbol{G}_{q u} \boldsymbol{U}=-\boldsymbol{G}_{q u} \overline{\boldsymbol{U}}
$$

Replacing in (47):

$$
-\boldsymbol{K}_{u q} \boldsymbol{Q}-\boldsymbol{G}_{u \sigma}\left[N_{0} \boldsymbol{K}_{\sigma \sigma}^{-1}\left(\boldsymbol{G}_{\sigma u} \boldsymbol{U}-\boldsymbol{G}_{\sigma u} \overline{\boldsymbol{U}}\right)-\boldsymbol{K}_{\sigma \sigma}^{-1} \boldsymbol{K}_{\sigma q} \boldsymbol{Q}\right]=\boldsymbol{F}_{u} .
$$

Again making use of:

$$
\boldsymbol{G}_{u \sigma} \boldsymbol{K}_{\sigma \sigma}^{-1} \boldsymbol{K}_{\sigma q} \boldsymbol{Q}=\boldsymbol{G}_{u q} \boldsymbol{Q},
$$

equation (47) turns into:

$$
-\boldsymbol{K}_{u q} \boldsymbol{Q}+\boldsymbol{G}_{u q} \boldsymbol{Q}-N_{0} \boldsymbol{G}_{u \sigma} \boldsymbol{K}_{\sigma \sigma}^{-1} \boldsymbol{G}_{\sigma u} \boldsymbol{U}=\boldsymbol{F}_{u}-N_{0} \boldsymbol{G}_{u \sigma} \boldsymbol{K}_{\sigma \sigma}^{-1} \boldsymbol{G}_{\sigma u} \overline{\boldsymbol{U}}
$$

The final variational form of the problem after condensation of the Lagrange multipliers is: find $\left[\boldsymbol{q}_{h}, u_{h}\right] \in$ $R_{h} \times V_{h}$ such that

$$
\begin{aligned}
-\frac{1}{k}\left(\boldsymbol{q}_{h}, \delta \boldsymbol{q}_{h}\right)_{\Omega}-\left(\nabla u_{h}, \delta \boldsymbol{q}_{h}\right)_{\Omega}-\left\langle\boldsymbol{n} \cdot \delta \boldsymbol{q}_{h}, u_{h}\right\rangle_{\Gamma} & =-\left\langle\delta \boldsymbol{q}_{n, h}, \bar{u}\right\rangle_{\Gamma}, \\
-\left(\nabla \delta u_{h}, \boldsymbol{q}_{h}\right)_{\Omega}-<\boldsymbol{n} \cdot \boldsymbol{q}_{h}, \delta u_{h}>_{\Gamma}-N_{0} c\left(\delta u_{h}, u_{h}\right) & =\left\langle f, \delta u_{h}\right\rangle_{\Omega}-N_{0} c\left(\delta u_{h}, \bar{u}\right),
\end{aligned}
$$

where the algebraic counterpart of $c\left(\delta u_{h}, u_{h}\right)$ is $\boldsymbol{G}_{u \sigma} \boldsymbol{K}_{\sigma \sigma}^{-1} \boldsymbol{G}_{\sigma u} \boldsymbol{U}$. Note that since $\Sigma_{h}$ is elementwise discontinuous, $\boldsymbol{K}_{\sigma \sigma}^{-1}$ can be computed locally at the element level. Note also that the only difference with respect to Nitsche's method is in the definition of $c\left(\delta u_{h}, u_{h}\right)$, which would be a boundary mass matrix in the case of Nitsche's method.

\subsection{Dual form of Darcy's problem}

The same equations (27)-(28) can be understood (in the sense of distributions) in a different functional setting to the one analyzed above. We may consider less regularity for $u$ and more regularity for $\boldsymbol{q}$. In particular, the problem is also well posed if $u \in L^{2}(\Omega)$ and $\boldsymbol{q} \in H(\operatorname{div}, \Omega)$, the space of functions in $L^{2}(\Omega)$ with divergence in $L^{2}(\Omega)$. In this case, the traces of $u$ are not defined on $\Gamma$, but the normal component of $\boldsymbol{q}$ has a well defined trace. Thus, the problem we consider now consists of finding $u$ and $\boldsymbol{q}$ such that

$$
\begin{aligned}
\frac{1}{k} \boldsymbol{q}+\nabla u & =\mathbf{0} & & \text { in } \Omega, \\
\nabla \cdot \boldsymbol{q} & =f & & \text { in } \Omega, \\
\boldsymbol{n} \cdot \boldsymbol{q} & =\bar{q}_{n} & & \text { on } \Gamma .
\end{aligned}
$$


In this case the problem has a functional framework different to the Poisson problem (16)-(17). Note also that $u$ is defined up to constants, i.e., $u \in L^{2}(\Omega) / \mathbb{R}$.

Using the notation introduced in Section $2, n=d+1, \mathcal{L}([\boldsymbol{q}, u])=\left[\frac{1}{k} \boldsymbol{q}+\nabla u, \nabla \cdot \boldsymbol{q}\right], \mathcal{D}([\boldsymbol{q}, u])=\boldsymbol{n} \cdot \boldsymbol{q}$ and

$$
\begin{aligned}
F([\boldsymbol{r}, v]) & =-\frac{1}{2 k}\|\boldsymbol{r}\|_{L^{2}(\Omega)}^{2}+(v, \nabla \cdot \boldsymbol{r})_{\Omega}-\langle f, v\rangle_{\Omega} \\
B([\boldsymbol{q}, u],[\delta \boldsymbol{q}, \delta u]) & =-\frac{1}{k}(\boldsymbol{q}, \delta \boldsymbol{q})_{\Omega}+(u, \nabla \cdot \delta \boldsymbol{q})_{\Omega}+(\delta u, \nabla \cdot \boldsymbol{q})_{\Omega} \\
L([\delta \boldsymbol{q}, \delta u]) & =\langle f, \delta u\rangle_{\Omega} .
\end{aligned}
$$

In this problem, the unknown with essential boundary conditions is itself a flux, and so we link it to the Lagrange multiplier $\mu$ by imposing that the discrete approximation to $\boldsymbol{q}$ be equal to the flux of the approximation of the Lagrange multiplier in a least squares sense.

As before, $N=N_{0} k$, with $N_{0}$ dimensionless. The problem is well posed in $X=H(\operatorname{div}, \Omega) \times L^{2}(\Omega)$, the space of traces being $\Lambda=H^{-1 / 2}(\Gamma)$ (the trace operator from $X$ to $\Lambda$ is $\left.[\boldsymbol{r}, v] \mapsto \boldsymbol{n} \cdot \boldsymbol{r}\right|_{\Gamma}$ ).

\subsubsection{Galerkin finite element approximation}

Let $V_{h} \subset L^{2}(\Omega) / \mathbb{R}$ be the finite element space to approximate $u, R_{h} \subset H\left(\operatorname{div}, \Omega_{h}\right)$ the finite element space to approximate $\boldsymbol{q}$ and $\Sigma_{h}$ the space to approximate the scalar whose flux allows one to prescribe in a weak form (53).

Following the ideas of Section 2, the method we propose consists in optimizing the functional

$$
\hat{G}\left(\left[\boldsymbol{r}_{h}, v_{h}, \eta_{h}\right]\right)=F\left(\left[\boldsymbol{r}_{h}, v_{h}\right]\right)-\left\langle\eta_{h}, \boldsymbol{n} \cdot \boldsymbol{r}_{h}-\bar{q}_{n}\right\rangle_{\Gamma}+\frac{1}{2 N_{0} k}\left\|k \nabla \eta_{h}+\boldsymbol{r}_{h}\right\|_{L^{2}(\Omega)}^{2}+\frac{\gamma k}{2 N_{0} L_{0}^{2}}\left\|\eta_{h}-v_{h}\right\|_{L^{2}(\Omega)}^{2},
$$

over $R_{h} \times V_{h} \times \Sigma_{h}$, where $\gamma>0$ and $L_{0}$ is a characteristic length of problem, which will be required at several instances for dimensional consistency. The need for the last term is to prove stability of the Galerkin approximation, and will be clear in the proof of Theorem 4 below. It can be observed that for the critical point of $\hat{G}$ the third term links the new Lagrange-multiplier-type variable to the flux, whereas the fourth term links it to the primal variable, in both cases in a least-squares sense.

Problem (14)-(15) now reads: find $\left[\boldsymbol{q}_{h}, u_{h}, \mu_{h}\right] \in R_{h} \times V_{h} \times \Sigma_{h}$ such that

$$
\begin{gathered}
-\frac{1}{k}\left(\boldsymbol{q}_{h}, \delta \boldsymbol{q}_{h}\right)_{\Omega}+\left(u_{h}, \nabla \cdot \delta \boldsymbol{q}_{h}\right)_{\Omega}-\left\langle\mu_{h}, \boldsymbol{n} \cdot \delta \boldsymbol{q}_{h}\right\rangle_{\Gamma}+\frac{1}{N_{0} k}\left(\delta \boldsymbol{q}_{h}, k \nabla \mu_{h}+\boldsymbol{q}_{h}\right)_{\Omega}=0, \\
\left(\delta u_{h}, \nabla \cdot \boldsymbol{q}_{h}\right)_{\Omega}-\frac{\gamma k}{N_{0} L_{0}^{2}}\left(\delta u_{h}, \mu_{h}-u_{h}\right)_{\Omega}=\left\langle f, \delta u_{h}\right\rangle_{\Omega}, \\
-\left\langle\delta \mu_{h}, \boldsymbol{n} \cdot \boldsymbol{q}_{h}\right\rangle_{\Gamma}+\frac{1}{N_{0} k}\left(k \nabla \delta \mu_{h}, k \nabla \mu_{h}+\boldsymbol{q}_{h}\right)_{\Omega}+\frac{\gamma k}{N_{0} L_{0}^{2}}\left(\delta \mu_{h}, \mu_{h}-u_{h}\right)_{\Omega}=-\left\langle\delta \mu_{h}, \bar{q}_{n}\right\rangle_{\Gamma},
\end{gathered}
$$

for all $\delta \boldsymbol{q}_{h} \in R_{h}, \delta u_{h} \in V_{h}$ and $\delta \mu_{h} \in \Sigma_{h}$. This is the formulation we propose to approximate in a weak sense boundary condition (53) for Darcy's problem in dual form and using the Galerkin finite element approximation. Contrary to the previous cases, the space of the new unknown introduced, $\Sigma_{h}$, needs to be made of continuous functions (or otherwise inter-element jumps need to be introduced, in a discontinuous Galerkin way). Therefore, (56) does not allow one to eliminate the degrees of freedom of $\mu_{h}$ at the element level. In this case, the benefit of the present method compared to a standard Lagrange multiplier technique is not the reduction of the number of degrees of freedom, but only to deal with unknowns defined on the whole computational domain rather that with unknowns defined only on $\Gamma$, as Lagrange multipliers would be.

In order to prove that the formulation proposed is stable, we will need two compatibility conditions. The first one is the 'dual' version of (23)-(24), and read as follows:

$$
\begin{aligned}
& \forall \boldsymbol{r}_{h} \in R_{h} \quad \exists \eta_{h} \in \Sigma_{h}, \delta_{0}>0 \text { such that }\left\|\boldsymbol{n} \cdot \boldsymbol{r}_{h}\right\|_{L^{2}(\Gamma)}^{2} \lesssim\left\langle\eta_{h}, \boldsymbol{n} \cdot \boldsymbol{r}_{h}\right\rangle_{\Gamma}+\delta_{0} h\left\|\nabla \cdot \boldsymbol{r}_{h}\right\|_{L^{2}(\Omega)}^{2}, \\
& \left\|\eta_{h}\right\|_{L^{2}(\Gamma)}=\left\|\boldsymbol{n} \cdot \boldsymbol{r}_{h}\right\|_{L^{2}(\Gamma)}, \quad\left\|\nabla \eta_{h}\right\|_{L^{2}(\Omega)}^{2} \lesssim \frac{h}{L_{0}^{2}}\left\|\boldsymbol{n} \cdot \boldsymbol{r}_{h}\right\|_{L^{2}(\Gamma)}^{2} .
\end{aligned}
$$

Note that we have used again the length scale $L_{0}$. Changing this length scale only implies a change in the constant involved in (58). As for (23)-(24), it can be shown that this condition holds in the case of equal order interpolation 
to construct $R_{h}$ and $\Sigma_{h}$, which in this problem is the only interesting case (recall that $\mu_{h}$ in (54)-(56) cannot be condensed).

The second condition we require is the well posedness of Darcy's problem when boundary conditions are imposed in the classical way, which in turn can be split into two conditions. First, we require that:

$$
\begin{aligned}
& \forall v_{h} \in V_{h} \quad \exists \boldsymbol{r}_{h} \in R_{h} \backslash\{\mathbf{0}\}, \boldsymbol{n} \cdot \boldsymbol{r}_{h}=0 \text { on } \partial \Omega_{h}, \text { such that } \\
& \beta\left\|v_{h}\right\|_{L^{2}(\Omega)}\left\|\boldsymbol{r}_{h}\right\|_{H(\operatorname{div}, \Omega)} \leq\left(v_{h}, \nabla \cdot \boldsymbol{r}_{h}\right)_{\Omega},
\end{aligned}
$$

for a constant $\beta>0$. Again, we have assumed this condition to hold when integrals are extended over $\Omega$, not over $\Omega_{h}$. The last case would require a minor modification in the proof of stability (see the proof of Theorem 6 below).

The second requirement is that the term $\left(\boldsymbol{q}_{h}, \delta \boldsymbol{q}_{h}\right)_{\Omega}$ understood as a bilinear form in $R_{h} \times R_{h}$ should be coercive in $Z_{h}=\left\{\boldsymbol{r}_{h} \in R_{h} \mid\left(v_{h}, \nabla \cdot \boldsymbol{r}_{h}\right)_{\Omega}=0 \forall v_{h} \in V_{h}\right\}$ with respect to the norm in $H(\operatorname{div}, \Omega)$. If $P_{V_{h}}$ is the $L^{2}$ projection onto $V_{h}$ and $P_{V_{h}}^{\perp}=I-P_{V_{h}}$, we may write $Z_{h}=\left\{\boldsymbol{r}_{h} \in R_{h} \mid P_{V_{h}}\left(\nabla \cdot \boldsymbol{r}_{h}\right)=0\right\}$. Since $\left(\boldsymbol{q}_{h}, \boldsymbol{q}_{h}\right)_{\Omega}=\left\|\boldsymbol{q}_{h}\right\|_{L^{2}(\Omega)}^{2}$, requiring coercivity of $\left(\boldsymbol{q}_{h}, \delta \boldsymbol{q}_{h}\right)_{\Omega}$ in $Z_{h}$ amounts to say that:

$$
\forall \boldsymbol{r}_{h} \in R_{h} \quad P_{V_{h}}^{\perp}\left(\nabla \cdot \boldsymbol{r}_{h}\right)=0 \Longleftrightarrow P_{V_{h}}\left(\nabla \cdot \boldsymbol{r}_{h}\right)=\nabla \cdot \boldsymbol{r}_{h}
$$

Examples of pairs $V_{h}-R_{h}$ that satisfy conditions (59) and (60) are the Raviart-Thomas or the Brezzi-DouglasMarini elements (see [5], for example).

The bilinear form associated to problem (54)-(56) is given by

$$
\begin{aligned}
B_{\mathrm{DDG}} & \left(\left[\boldsymbol{q}_{h}, u_{h}, \mu_{h}\right],\left[\delta \boldsymbol{q}_{h}, \delta u_{h}, \delta \mu_{h}\right]\right)=-\frac{1}{k}\left(\boldsymbol{q}_{h}, \delta \boldsymbol{q}_{h}\right)_{\Omega}+\left(u_{h}, \nabla \cdot \delta \boldsymbol{q}_{h}\right)_{\Omega}+\left(\delta u_{h}, \nabla \cdot \boldsymbol{q}_{h}\right)_{\Omega} \\
& -\left\langle\delta \mu_{h}, \boldsymbol{n} \cdot \boldsymbol{q}_{h}\right\rangle_{\Gamma}-\left\langle\mu_{h}, \boldsymbol{n} \cdot \delta \boldsymbol{q}_{h}\right\rangle_{\Gamma} \\
& +\frac{1}{N_{0} k}\left(k \nabla \delta \mu_{h}+\delta \boldsymbol{q}_{h}, k \nabla \mu_{h}+\boldsymbol{q}_{h}\right)_{\Omega}+\frac{\gamma k}{N_{0} L_{0}^{2}}\left(\delta \mu_{h}-\delta u_{h}, \mu_{h}-u_{h}\right)_{\Omega} .
\end{aligned}
$$

We next prove that it is stable in the norm

$$
\begin{aligned}
\left\|\left[\boldsymbol{q}_{h}, u_{h}, \mu_{h}\right]\right\|_{\mathrm{DDG}}^{2} & :=\frac{1}{k}\left\|\boldsymbol{q}_{h}\right\|_{L^{2}(\Omega)}^{2}+\frac{L_{0}^{2}}{k}\left\|\nabla \cdot \boldsymbol{q}_{h}\right\|_{L^{2}(\Omega)}^{2}+\frac{L_{0}^{2}}{k h}\left\|\boldsymbol{n} \cdot \boldsymbol{q}_{h}\right\|_{L^{2}(\Gamma)}^{2} \\
& +\frac{k}{L_{0}^{2}}\left\|u_{h}\right\|_{L^{2}(\Omega)}^{2}+k\left\|\nabla \mu_{h}\right\|_{L^{2}(\Omega)}^{2}+\frac{\gamma k}{L_{0}^{2}}\left\|\mu_{h}\right\|_{L^{2}(\Omega)}^{2} .
\end{aligned}
$$

Theorem 4. Assume that (57)-(58) hold, that $N_{0}>1, \gamma>0$ and that (59)-(60) also hold. Then, if $h$ is small enough, for all $\left[\boldsymbol{q}_{h}, u_{h}, \mu_{h}\right]$ there exists a non trivial $\left[\delta \boldsymbol{q}_{h}, \delta u_{h}, \delta \mu_{h}\right]$ such that:

$$
B_{\mathrm{DDG}}\left(\left[\boldsymbol{q}_{h}, u_{h}, \mu_{h}\right],\left[\delta \boldsymbol{q}_{h}, \delta u_{h}, \delta \mu_{h}\right]\right) \gtrsim\left\|\left[\boldsymbol{q}_{h}, u_{h}, \mu_{h}\right]\right\|_{\mathrm{DDG}}\left\|\left[\delta \boldsymbol{q}_{h}, \delta u_{h}, \delta \mu_{h}\right]\right\|_{\mathrm{DDG}} \cdot
$$

Proof. Let us start noting that, for $N_{0}>1$,

$$
B_{\mathrm{DDG}}\left(\left[\boldsymbol{q}_{h}, u_{h}, \mu_{h}\right],\left[-\boldsymbol{q}_{h}, u_{h}, \mu_{h}\right]\right) \gtrsim \frac{1}{k}\left\|\boldsymbol{q}_{h}\right\|_{L^{2}(\Omega)}^{2}+k\left\|\nabla \mu_{h}\right\|_{L^{2}(\Omega)}^{2}+\frac{\gamma k}{L_{0}^{2}}\left\|\mu_{h}-u_{h}\right\|_{L^{2}(\Omega)}^{2} .
$$

Condition (60) allows us to take $\delta u_{h}=k^{-1} L_{0}^{2} \nabla \cdot \boldsymbol{q}_{h}$ and get

$$
B_{\mathrm{DDG}}\left(\left[\boldsymbol{q}_{h}, u_{h}, \mu_{h}\right],\left[\mathbf{0}, k^{-1} L_{0}^{2} \nabla \cdot \boldsymbol{q}_{h}, 0\right]\right) \gtrsim \frac{L_{0}^{2}}{k}\left\|\nabla \cdot \boldsymbol{q}_{h}\right\|_{L^{2}(\Omega)}^{2}-\frac{\gamma k}{L_{0}^{2}}\left\|\mu_{h}-u_{h}\right\|_{L^{2}(\Omega)}^{2} .
$$

Let $\boldsymbol{r}_{h}^{u}$ be the function that allows one to guarantee (59) for $v_{h}=u_{h}$, normalized so that

$$
\left\|\boldsymbol{r}_{h}^{u}\right\|_{H(\operatorname{div}, \Omega)}:=\left\|\nabla \cdot \boldsymbol{r}_{h}^{u}\right\|_{L^{2}(\Omega)}+\frac{1}{L_{0}}\left\|\boldsymbol{r}_{h}^{u}\right\|_{L^{2}(\Omega)}=\left\|u_{h}\right\|_{L^{2}(\Omega)} .
$$


It is easily checked that

$$
B_{\mathrm{DDG}}\left(\left[\boldsymbol{q}_{h}, u_{h}, \boldsymbol{\sigma}_{h}\right],\left[k L_{0}^{-2} \boldsymbol{r}_{h}^{u}, 0, \mathbf{0}\right]\right) \gtrsim \frac{k}{L_{0}^{2}}\left\|u_{h}\right\|_{L^{2}(\Omega)}^{2}-\frac{1}{k}\left\|\boldsymbol{q}_{h}\right\|_{L^{2}(\Omega)}^{2}-k\left\|\nabla \mu_{h}\right\|_{L^{2}(\Omega)}^{2}-\frac{k}{L_{0}^{2}}\left\langle\mu_{h}, \boldsymbol{n} \cdot \boldsymbol{r}_{h}^{u}\right\rangle_{\Gamma} .
$$

Let us elaborate on the last term. Since $\partial \Omega_{\Gamma, \text { out }}=\partial \Omega_{h} \cup \Gamma$ and $\boldsymbol{n} \cdot \boldsymbol{r}_{h}^{u}=0$ on $\partial \Omega_{h}$, with the normal to $\partial \Omega_{\Gamma \text {,out }}$ opposite to the normal to $\Gamma$, we have that

$$
\frac{k}{L_{0}^{2}}\left\langle\mu_{h}, \boldsymbol{n} \cdot \boldsymbol{r}_{h}^{u}\right\rangle_{\Gamma}=-\frac{k}{L_{0}^{2}} \int_{\partial \Omega_{\Gamma, \text { out }}} \mu_{h} \boldsymbol{n} \cdot \boldsymbol{r}_{h}^{u}=-\frac{k}{L_{0}^{2}} \int_{\Omega_{\Gamma, \text { out }}}\left(\mu_{h} \nabla \cdot \boldsymbol{r}_{h}^{u}+\nabla \mu_{h} \cdot \boldsymbol{r}_{h}^{u}\right) .
$$

Since the measure of $\Omega_{\Gamma, \text { out }}$ tends to zero as $h \rightarrow 0$, we may write

$$
\frac{k}{L_{0}^{2}}\left\langle\mu_{h}, \boldsymbol{n} \cdot \boldsymbol{r}_{h}^{u}\right\rangle_{\Gamma} \lesssim \frac{k}{L_{0}^{2}} \varphi(h)\left\|\mu_{h}\right\|_{L^{2}(\Omega)}\left\|u_{h}\right\|_{L^{2}(\Omega)}+\frac{k}{L_{0}} \varphi(h)\left\|\nabla \mu_{h}\right\|_{L^{2}(\Omega)}\left\|u_{h}\right\|_{L^{2}(\Omega)},
$$

with $\varphi(h) \rightarrow 0$ as $h \rightarrow 0$. The norms on the right-hand-side can be understood as normalizing factors. Therefore

$$
\begin{aligned}
B_{\mathrm{DDG}}\left(\left[\boldsymbol{q}_{h}, u_{h}, \boldsymbol{\sigma}_{h}\right],\left[k L_{0}^{-2} \boldsymbol{r}_{h}^{u}, 0, \mathbf{0}\right]\right) & \gtrsim \frac{k}{L_{0}^{2}}\left\|u_{h}\right\|_{L^{2}(\Omega)}^{2}-\frac{1}{k}\left\|\boldsymbol{q}_{h}\right\|_{L^{2}(\Omega)}^{2} \\
& -k\left\|\nabla \mu_{h}\right\|_{L^{2}(\Omega)}^{2}-\frac{k}{L_{0}^{2}} \varphi(h)\left\|\mu_{h}\right\|_{L^{2}(\Omega)} .
\end{aligned}
$$

From (63), (64) and (65) it follows that if $\left[\delta \boldsymbol{q}_{h}, \delta u_{h}, \delta \mu_{h}\right]=\left[-\boldsymbol{q}_{h}+\beta k L_{0}^{-2} \boldsymbol{r}_{h}^{u}, u_{h}+k^{-1} L_{0}^{2} \nabla \cdot \boldsymbol{q}_{h}, \mu_{h}\right]$, for an appropriate $\beta$,

$$
\begin{aligned}
B_{\mathrm{DDG}}\left(\left[\boldsymbol{q}_{h}, u_{h}, \boldsymbol{\sigma}_{h}\right],\left[\delta \boldsymbol{q}_{h}, \delta u_{h}, \delta \mu_{h}\right]\right) & \gtrsim \frac{1}{k}\left\|\boldsymbol{q}_{h}\right\|_{L^{2}(\Omega)}^{2}+\frac{L_{0}^{2}}{k}\left\|\nabla \cdot \boldsymbol{q}_{h}\right\|_{L^{2}(\Omega)}^{2} \\
& +\frac{k}{L_{0}^{2}}\left\|u_{h}\right\|_{L^{2}(\Omega)}^{2}+k\left\|\nabla \mu_{h}\right\|_{L^{2}(\Omega)}^{2}+\frac{\gamma k}{L_{0}^{2}}\left\|\mu_{h}\right\|_{L^{2}(\Omega)}^{2},
\end{aligned}
$$

for $h$ sufficiently small. It is observed from (65) that we have to have some control of $\left\|\mu_{h}\right\|_{L^{2}(\Omega)}$, and this is why we have introduced the term multiplied by $\gamma>0$ in (61).

It remains to control the boundary term in (62), which can be done as in the previous cases using (57)-(58). It $\eta_{h}^{q} \in \Sigma_{h}$ is the function that allows one to guarantee (57) for $\boldsymbol{r}_{h}=\boldsymbol{q}_{h}$, it can be verified that

$$
B_{\mathrm{DDG}}\left(\left[\boldsymbol{q}_{h}, u_{h}, \boldsymbol{\sigma}_{h}\right],\left[\delta \boldsymbol{q}_{h}, \delta u_{h}, \delta \mu_{h}+\beta^{\prime} \eta_{h}^{q}\right]\right) \gtrsim\left\|\left[\boldsymbol{q}_{h}, u_{h}, \mu_{h}\right]\right\|_{\mathrm{DDG}}^{2},
$$

for an appropriate $\beta^{\prime}$. The proof concludes checking that $\left\|\left[\boldsymbol{q}_{h}, u_{h}, \mu_{h}\right]\right\|_{\mathrm{DDG}} \gtrsim\left\|\left[\delta \boldsymbol{q}_{h}, \delta u_{h}, \delta \mu_{h}+\beta^{\prime} \eta_{h}^{q}\right]\right\|_{\mathrm{DDG}}$.

\subsubsection{Stabilized finite element approximation}

As for the primal form of Darcy's problem, the alternative to satisfy the compatibility condition (59) is to use a stabilized finite element formulation. Again, we refer to $[1,2]$ for background about the method we use.

The stabilizing terms to be added to the Galerkin formulation are the same as for the primal form of the problem. Therefore, the method reads: find $\left[\boldsymbol{q}_{h}, u_{h}, \boldsymbol{\sigma}_{h}\right] \in R_{h} \times V_{h} \times \Sigma_{h}$ such that

$$
B_{\mathrm{DDS}}\left(\left[\boldsymbol{q}_{h}, u_{h}, \mu_{h}\right],\left[\delta \boldsymbol{q}_{h}, \delta u_{h}, \delta \mu_{h}\right]\right)=L_{\mathrm{DDS}}\left(\left[\delta \boldsymbol{q}_{h}, \delta u_{h}, \delta \mu_{h}\right]\right),
$$

for all $\left[\delta \boldsymbol{q}_{h}, \delta u_{h}, \delta \mu_{h}\right] \in R_{h} \times V_{h} \times \Sigma_{h}$, where

$$
\begin{aligned}
B_{\mathrm{DDS}}\left(\left[\boldsymbol{q}_{h}, u_{h}, \mu_{h}\right],\left[\delta \boldsymbol{q}_{h}, \delta u_{h}, \delta \mu_{h}\right]\right) & =B_{\mathrm{DDG}}\left(\left[\boldsymbol{q}_{h}, u_{h}, \mu_{h}\right],\left[\delta \boldsymbol{q}_{h}, \delta u_{h}, \delta \mu_{h}\right]\right) \\
& +\sum_{K} \tau_{q}\left(\frac{1}{k} \delta \boldsymbol{q}_{h}+\nabla \delta u_{h}, \frac{1}{k} \boldsymbol{q}_{h}+\nabla u_{h}\right)_{K \cap \Omega} \\
& -\sum_{K} \tau_{u}\left(\nabla \cdot \boldsymbol{q}_{h}, \nabla \cdot \delta \boldsymbol{q}_{h}\right)_{K \cap \Omega} \\
L_{\mathrm{DDS}}\left(\left[\delta \boldsymbol{q}_{h}, \delta u_{h}, \delta \mu_{h}\right]\right)= & \left\langle f, \delta u_{h}\right\rangle_{\Omega}-\left\langle\delta \mu_{h}, \bar{q}_{n}\right\rangle_{\Gamma}-\sum_{K} \tau_{u}\left(\nabla \cdot \delta \boldsymbol{q}_{h}, f\right)_{K \cap \Omega},
\end{aligned}
$$


with $B_{\mathrm{DDG}}$ given by (61).

The stabilization parameters are different to those employed for the primal form of the problem. In order to get optimal stability and convergence for the dual problem they need to be computed as

$$
\tau_{q}=k \frac{h^{2}}{L_{0}^{2}} \tau_{q}^{0}, \quad \tau_{u}=\frac{L_{0}^{2}}{k} \tau_{u}^{0},
$$

with $\tau_{q}^{0}$ and $\tau_{u}^{0}$ dimensionless algorithmic constants.

We next show that the bilinear form $B_{\mathrm{DDS}}$ is stable in the norm

$$
\left\|\left[\boldsymbol{q}_{h}, u_{h}, \mu_{h}\right]\right\|_{\mathrm{DDS}}^{2}:=\left\|\left[\boldsymbol{q}_{h}, u_{h}, \mu_{h}\right]\right\|_{\mathrm{DDG}}^{2}+\tau_{q}^{0} k \frac{h^{2}}{L_{0}^{2}}\left\|\nabla u_{h}\right\|_{L^{2}(\Omega)}^{2} .
$$

where $\left\|\left[\boldsymbol{q}_{h}, u_{h}, \mu_{h}\right]\right\|_{\mathrm{DDG}}^{2}$ is defined in (62). The last term in (70) provides no significant control on the gradient of $u_{h}$, but appears if $\tau_{q}^{0}>0$. In this case we may safely take $\tau_{q}^{0}=0$.

Theorem 5. Assume that $N_{0}>1$ and that $0<\tau_{u}^{0}, 0 \leq \tau_{q}^{0}<1$ in (69). Then, for all $\left[\boldsymbol{q}_{h}, u_{h}, \mu_{h}\right]$ there exists a non trivial $\left[\delta \boldsymbol{q}_{h}, \delta u_{h}, \delta \mu_{h}\right]$ such that:

$$
B_{\mathrm{DDS}}\left(\left[\boldsymbol{q}_{h}, u_{h}, \mu_{h}\right],\left[\delta \boldsymbol{q}_{h}, \delta u_{h}, \delta \mu_{h}\right]\right) \gtrsim\left\|\left[\boldsymbol{q}_{h}, u_{h}, \mu_{h}\right]\right\|_{\mathrm{DDS}}\left\|\left[\delta \boldsymbol{q}_{h}, \delta u_{h}, \delta \mu_{h}\right]\right\|_{\mathrm{DDS}} .
$$

Proof. The proof is similar to that of Theorem 4. For $N_{0}>1$ and $\tau_{q}^{0}<1$ we get

$$
\begin{aligned}
B_{\mathrm{DDS}}\left(\left[\boldsymbol{q}_{h}, u_{h}, \mu_{h}\right],\left[-\boldsymbol{q}_{h}, u_{h}, \mu_{h}\right]\right) & \gtrsim \frac{1}{k}\left\|\boldsymbol{q}_{h}\right\|_{L^{2}(\Omega)}^{2}+k\left\|\nabla \mu_{h}\right\|_{L^{2}(\Omega)}^{2}+\frac{\gamma k}{L_{0}^{2}}\left\|\mu_{h}-u_{h}\right\|_{L^{2}(\Omega)}^{2} \\
& +\frac{L_{0}^{2}}{k}\left\|\nabla \cdot \boldsymbol{q}_{h}\right\|_{L^{2}(\Omega)}^{2}+\tau_{q}^{0} k \frac{h^{2}}{L_{0}^{2}}\left\|\nabla u_{h}\right\|_{L^{2}(\Omega)}^{2},
\end{aligned}
$$

so that now we do not need (60) to get control on the divergence of $\boldsymbol{q}_{h}$. To get control on the boundary norm of $\boldsymbol{q}_{h}$ we can proceed as in Theorem 4 . It only remains to get control on $\left\|u_{h}\right\|_{L^{2}(\Omega)}^{2}$. To this end, we can make use of the inf-sup condition that holds at the continuous level, as in [1], page 1981. We omit the technical details.

\subsubsection{Matrix structure}

Let us consider the simplified Galerkin weak form of the dual form of Darcy's problem: find $\left[\boldsymbol{q}_{h}, u_{h}, \mu_{h}\right] \in$ $R_{h} \times V_{h} \times \Sigma_{h}$ such that:

$$
\begin{aligned}
-\frac{1}{k}\left(\boldsymbol{q}_{h}, \delta \boldsymbol{q}_{h}\right)_{\Omega}+\left(u_{h}, \nabla \cdot \delta \boldsymbol{q}_{h}\right)_{\Omega}-\left\langle\mu_{h}, \boldsymbol{n} \cdot \delta \boldsymbol{q}_{h}\right\rangle_{\Gamma} & =0, \\
\left(\delta u_{h}, \nabla \cdot \boldsymbol{q}_{h}\right)_{\Omega}-\frac{\gamma k}{N_{0} L_{0}^{2}}\left(\delta u_{h}, \mu_{h}-u_{h}\right)_{\Omega} & =\left\langle f, \delta u_{h}\right\rangle_{\Omega}, \\
-\left\langle\delta \mu_{h}, \boldsymbol{n} \cdot \boldsymbol{q}_{h}\right\rangle_{\Gamma}+\frac{\gamma k}{N_{0} L_{0}^{2}}\left(\delta \mu_{h}, \mu_{h}-u_{h}\right)_{\Omega} & =-\left\langle\delta \mu_{h}, \bar{q}_{n}\right\rangle_{\Gamma},
\end{aligned}
$$

The problem in algebraic form is:

$$
\begin{aligned}
-\boldsymbol{K}_{q q} \boldsymbol{Q}+\hat{\boldsymbol{K}}_{q u} \boldsymbol{U}-\boldsymbol{G}_{q \mu} \boldsymbol{\mu} & =0, \\
\hat{\boldsymbol{K}}_{u q} \boldsymbol{Q}-\frac{1}{N_{0}} \boldsymbol{K}_{u \mu} \boldsymbol{\mu}+\frac{1}{N_{0}} \boldsymbol{K}_{u u} \boldsymbol{U} & =\boldsymbol{F}_{u}, \\
-\boldsymbol{G}_{\mu q} \boldsymbol{Q}+\frac{1}{N_{0}} \boldsymbol{K}_{\mu \mu} \boldsymbol{\mu}-\frac{1}{N_{0}} \boldsymbol{K}_{\mu u} \boldsymbol{U} & =-\boldsymbol{G}_{\mu q} \overline{\boldsymbol{Q}} .
\end{aligned}
$$

with the definition of each term given in Table 2 and Table 1. 


\begin{tabular}{|c|c|c|c|}
\hline$\hat{\boldsymbol{K}}_{q u} \boldsymbol{U}$ & $\boldsymbol{G}_{q \mu} \boldsymbol{\mu}$ & $\hat{\boldsymbol{K}}_{u q} \boldsymbol{Q}$ & $\boldsymbol{K}_{u \mu} \boldsymbol{\mu}$ \\
\hline \hline$\left(u_{h}, \nabla \cdot \delta \boldsymbol{q}_{h}\right)_{\Omega}$ & $\left\langle\mu_{h}, \boldsymbol{n} \cdot \delta \boldsymbol{q}_{h}\right\rangle_{\Gamma}$ & $\left(\delta u_{h}, \nabla \cdot \boldsymbol{q}_{h}\right)_{\Omega}$ & $\frac{\gamma k}{L_{0}^{2}}\left(\delta u_{h}, \mu_{h}\right)_{\Omega}$ \\
\hline \hline $\boldsymbol{K}_{u u} \boldsymbol{U}$ & $\boldsymbol{G}_{\mu q} \boldsymbol{Q}$ & $\boldsymbol{K}_{\mu \mu} \boldsymbol{\mu}$ & $\boldsymbol{K}_{\mu u} \boldsymbol{U}$ \\
\hline \hline$\frac{\gamma k}{L_{0}^{2}}\left(\delta u_{h}, u_{h}\right)_{\Omega}$ & $\left\langle\delta \mu_{h}, \boldsymbol{n} \cdot \boldsymbol{q}_{h}\right\rangle_{\Gamma}$ & $\frac{\gamma k}{L_{0}^{2}}\left(\delta \mu_{h}, \mu_{h}\right)_{\Omega}$ & $\frac{\gamma k}{L_{0}^{2}}\left(\delta \mu_{h}, u_{h}\right)_{\Omega}$ \\
\hline
\end{tabular}

Table 2: Algebraic terms for the dual form of Darcy's problem.

We choose $\Sigma_{h}$ such that $V_{h} \subset \Sigma_{h}$, and $\Sigma_{h}$ is elementwise discontinuous. From (76) we get:

$$
\begin{aligned}
\boldsymbol{G}_{\mu q} \boldsymbol{Q}+\frac{1}{N_{0}} \boldsymbol{K}_{\mu \mu} \boldsymbol{\mu}-\frac{1}{N_{0}} \boldsymbol{K}_{\mu u} \boldsymbol{U} & =\frac{1}{N_{0}} \boldsymbol{K}_{\mu q} \overline{\boldsymbol{Q}}, \\
\boldsymbol{\mu} & =N_{0} \boldsymbol{K}_{\mu \mu}^{-1}\left[\boldsymbol{G}_{\mu q} \overline{\boldsymbol{Q}}-\boldsymbol{G}_{\mu q} \boldsymbol{Q}\right]+\boldsymbol{K}_{\mu \mu}^{-1} \boldsymbol{K}_{\mu u} \boldsymbol{U} .
\end{aligned}
$$

Replacing in (76):

$$
-\boldsymbol{K}_{q q} \boldsymbol{Q}+\hat{\boldsymbol{K}}_{q u} \boldsymbol{U}-\boldsymbol{G}_{q \mu}\left(N_{0} \boldsymbol{K}_{\mu \mu}^{-1}\left[\boldsymbol{G}_{\mu q} \overline{\boldsymbol{Q}}-\boldsymbol{G}_{\mu q} \boldsymbol{Q}\right]+\boldsymbol{K}_{\mu \mu}^{-1} \boldsymbol{K}_{\mu u} \boldsymbol{U}\right)=0 .
$$

We note that, due to $V_{h} \subset \Sigma_{h}$ :

$$
\boldsymbol{G}_{q \mu} \boldsymbol{K}_{\mu \mu}^{-1} \boldsymbol{K}_{\mu u} \boldsymbol{U}=\boldsymbol{G}_{q u} \boldsymbol{U}
$$

and then (76) can be written as:

$$
-\boldsymbol{K}_{q q} \boldsymbol{Q}+\hat{\boldsymbol{K}}_{q u} \boldsymbol{U}-\boldsymbol{G}_{q u} \boldsymbol{U}-\boldsymbol{G}_{q \mu} N_{0} \boldsymbol{K}_{\mu \mu}^{-1}\left[\boldsymbol{G}_{\mu q} \overline{\boldsymbol{Q}}-\boldsymbol{G}_{\mu q} \boldsymbol{Q}\right]=0 .
$$

Replacing in (76):

$$
\hat{\boldsymbol{K}}_{u q} \boldsymbol{Q}-\frac{1}{N_{0}} \boldsymbol{K}_{u \mu}\left(N_{0} \boldsymbol{K}_{\mu \mu}^{-1}\left[\boldsymbol{G}_{\mu q} \overline{\boldsymbol{Q}}-\boldsymbol{G}_{\mu q} \boldsymbol{Q}\right]+\boldsymbol{K}_{\mu \mu}^{-1} \boldsymbol{K}_{\mu u} \boldsymbol{U}\right)+\frac{1}{N_{0}} \boldsymbol{K}_{u u} \boldsymbol{U}=\boldsymbol{F}_{u} .
$$

Again, due to $V_{h} \subset \Sigma_{h}$ :

$$
\begin{aligned}
\boldsymbol{K}_{u \mu} \boldsymbol{K}_{\mu \mu}^{-1} \boldsymbol{K}_{\mu u} \boldsymbol{U} & =\boldsymbol{K}_{u u} \boldsymbol{U} \\
\boldsymbol{K}_{u \mu} \boldsymbol{K}_{\mu \mu}^{-1} \boldsymbol{G}_{\mu q} \boldsymbol{Q} & =\boldsymbol{G}_{u q} \boldsymbol{Q},
\end{aligned}
$$

and then (76) can be written as:

$$
\hat{\boldsymbol{K}}_{u q} \boldsymbol{Q}-\left[\boldsymbol{G}_{u q} \overline{\boldsymbol{Q}}-\boldsymbol{G}_{u q} \boldsymbol{Q}\right]=\boldsymbol{F}_{u} .
$$

The final variational form of the problem after condensation of the Lagrange multipliers is: find $\left[\boldsymbol{q}_{h}, u_{h}\right] \in$ $R_{h} \times V_{h}$ such that:

$$
\begin{aligned}
-\frac{1}{k}\left(\boldsymbol{q}_{h}, \delta \boldsymbol{q}_{h}\right)_{\Omega}+\left(u_{h}, \nabla \cdot \delta \boldsymbol{q}_{h}\right)_{\Omega}-\left\langle u_{h}, \boldsymbol{n} \cdot \delta \boldsymbol{q}_{h}\right\rangle_{\Gamma}-N_{0} d\left(\boldsymbol{q}_{h}, \delta \boldsymbol{q}_{h}\right) & =-N_{0} d\left(\overline{\boldsymbol{q}}, \delta \boldsymbol{q}_{h}\right), \\
\left(\delta u_{h}, \nabla \cdot \boldsymbol{q}_{h}\right)_{\Omega}+\left\langle\boldsymbol{n} \cdot \boldsymbol{q}_{h}, \delta u_{h}\right\rangle_{\Gamma} & =\left\langle f, \delta u_{h}\right\rangle_{\Omega}+\left\langle\boldsymbol{n} \cdot \overline{\boldsymbol{q}}, \delta u_{h}\right\rangle_{\Gamma},
\end{aligned}
$$

where the algebraic counterpart of $d\left(\boldsymbol{q}_{h}, \delta \boldsymbol{q}_{h}\right)$ is $\boldsymbol{G}_{q \mu} \boldsymbol{K}_{\mu \mu}^{-1} \boldsymbol{G}_{\mu q} \boldsymbol{Q}$. Again, the only difference with respect to a standard application of Nitsche's method to the dual form of Darcy's problem is in the definition of $d\left(\boldsymbol{q}_{h}, \delta \boldsymbol{q}_{h}\right)$, which would consist in a boundary mass matrix times a penalty parameter in Nitsche's method. 


\section{Stokes' problem}

In this section we consider a third example of elliptic problem, namely, the Stokes problem for an incompressible material. To fix ideas, we will use the terminology of fluid mechanics to refer to the unknowns and the physical properties. The techniques of Section 2 can be directly applied with the appropriate identification of the unknowns, operators and functional spaces in play.

For completeness, we will consider two versions of the problem. We will start with the most classical twofield approach, involving only velocities and pressures as variables, and then we will describe how to deal with the three-field approach, where deviatoric stresses are considered as an independent variable. In both cases, we shall describe the Galerkin and a stabilized finite element formulation.

\subsection{Two-field approach}

Let $\boldsymbol{u}: \Omega \longrightarrow \mathbb{R}^{d}$ be the velocity and $p: \Omega \longrightarrow \mathbb{R}$ the pressure of a fluid moving in $\Omega$ of (kinematic) viscosity $\nu$ and subject to a body force $\boldsymbol{f}$. The Stokes problem consists of finding $\boldsymbol{u}$ and $p$ as the solution to the boundary value problem

$$
\begin{aligned}
-2 \nu \nabla \cdot \nabla^{S} \boldsymbol{u}+\nabla p & =\boldsymbol{f} & & \text { in } \Omega \\
\nabla \cdot \boldsymbol{u} & =0 & & \text { in } \Omega \\
\boldsymbol{u} & =\overline{\boldsymbol{u}} & & \text { on } \Gamma
\end{aligned}
$$

where $\overline{\boldsymbol{u}}$ is a given velocity prescribed on $\Gamma$ and $\nabla^{S} \boldsymbol{u}$ stands for the symmetrical part of $\nabla \boldsymbol{u}$.

Using the notation of Section 2 and organizing the unknowns as $[\boldsymbol{u}, p]$, now we have that $n=d+1$, $\mathcal{L}([\boldsymbol{u}, p])=[-\nu \Delta \boldsymbol{u}+\nabla p, \nabla \cdot \boldsymbol{u}], \mathcal{D}([\boldsymbol{u}, p])=\boldsymbol{u}$, and

$$
\begin{aligned}
F([\boldsymbol{v}, q]) & =\nu\left\|\nabla^{S} \boldsymbol{v}\right\|_{L^{2}(\Omega)}^{2}-(q, \nabla \cdot \boldsymbol{v})_{\Omega}-\langle\boldsymbol{f}, \boldsymbol{v}\rangle_{\Omega} \\
B([\boldsymbol{u}, p],[\delta \boldsymbol{u}, \delta p]) & =2 \nu\left(\nabla^{S} \boldsymbol{u}, \nabla^{S} \delta \boldsymbol{u}\right)_{\Omega}-(p, \nabla \cdot \delta \boldsymbol{u})_{\Omega}-(\delta p, \nabla \cdot \boldsymbol{u})_{\Omega} \\
\mathcal{F}([\boldsymbol{u}, p]) & =-p \boldsymbol{I}+2 \nu \nabla^{S} \boldsymbol{u}
\end{aligned}
$$

where $\boldsymbol{I}$ is the identity in $\mathbb{R}^{d}$. We now choose $N=N_{0} \nu$, with $N_{0}$ dimensionless. The problem is well posed in the space $X=V \times Q=H^{1}(\Omega)^{d} \times L^{2}(\Omega) / \mathbb{R}$, and the space of traces is $\Lambda=H^{1 / 2}(\Gamma)^{d}$, the trace operator being $\left.[\boldsymbol{u}, p] \mapsto \boldsymbol{u}\right|_{\Gamma}$

\subsubsection{Galerkin finite element approximation}

Let $V_{h} \subset V$ and $Q_{h} \subset Q$ be the finite element spaces constructed from the finite element partition to approximate $V$ and $Q$, respectively, and let $\Sigma_{h}$ be the space of the new variable to be introduced for enforcing (79) weakly. Using the ideas presented in Section 2, the method we propose consists of optimizing the functional

$$
\hat{G}\left(\left[\boldsymbol{v}_{h}, q_{h}, \boldsymbol{\xi}_{h}\right]\right)=F\left(\left[\boldsymbol{v}_{h}, q_{h}\right]\right)-\left\langle\boldsymbol{n} \cdot \boldsymbol{\xi}_{h}, \boldsymbol{v}_{h}-\overline{\boldsymbol{u}}\right\rangle_{\Gamma}+\left\langle q_{h}, \boldsymbol{n} \cdot \boldsymbol{v}_{h}-\boldsymbol{n} \cdot \overline{\boldsymbol{u}}\right\rangle_{\Gamma}-\frac{1}{4 N_{0} \nu}\left\|\boldsymbol{\xi}_{h}-2 \nu \nabla^{S} \boldsymbol{v}_{h}\right\|_{L^{2}(\Omega)}^{2},
$$

on $V_{h} \times Q_{h} \times \Sigma_{h}$, and problem (14)-(15) in this case reads: find $\left[\boldsymbol{u}_{h}, p_{h}, \boldsymbol{\sigma}_{h}\right] \in V_{h} \times Q_{h} \times \Sigma_{h}$ such that

$$
\begin{aligned}
& 2 \nu\left(\nabla^{S} \boldsymbol{u}_{h}, \nabla^{S} \delta \boldsymbol{u}_{h}\right)_{\Omega}-\left(p_{h}, \nabla \cdot \delta \boldsymbol{u}_{h}\right)_{\Omega}-\left\langle\boldsymbol{n} \cdot \boldsymbol{\sigma}_{h}, \delta \boldsymbol{u}_{h}\right\rangle_{\Gamma}+\left\langle p_{h}, \boldsymbol{n} \cdot \delta \boldsymbol{u}_{h}\right\rangle_{\Gamma} \\
&-\frac{1}{2 N_{0} \nu}\left(-2 \nu \nabla^{S} \delta \boldsymbol{u}_{h}, \boldsymbol{\sigma}_{h}-2 \nu \nabla^{S} \boldsymbol{u}_{h}\right)_{\Omega}=\left\langle\boldsymbol{f}, \delta \boldsymbol{u}_{h}\right\rangle_{\Omega}, \\
&-\left(\delta p_{h}, \nabla \cdot \boldsymbol{u}_{h}\right)_{\Omega}+\left\langle\delta p_{h}, \boldsymbol{n} \cdot \boldsymbol{u}_{h}\right\rangle_{\Gamma}=\left\langle\delta p_{h}, \boldsymbol{n} \cdot \overline{\boldsymbol{u}}\right\rangle_{\Gamma}, \\
&-\left\langle\boldsymbol{n} \cdot \delta \boldsymbol{\sigma}_{h}, \boldsymbol{u}_{h}\right\rangle_{\Gamma}-\frac{1}{2 N_{0} \nu}\left(\delta \boldsymbol{\sigma}_{h}, \boldsymbol{\sigma}_{h}-2 \nu \nabla^{S} \boldsymbol{u}_{h}\right)_{\Omega}=-\left\langle\boldsymbol{n} \cdot \delta \boldsymbol{\sigma}_{h}, \overline{\boldsymbol{u}}\right\rangle_{\Gamma},
\end{aligned}
$$

for all $\left[\delta \boldsymbol{u}_{h}, \delta p_{h}, \delta \boldsymbol{\sigma}_{h}\right] \in V_{h} \times Q_{h} \times \Sigma_{h}$. Even if it is not our intention to analyze convergence of this method, but only stability, let us remark a key ingredient for the numerical solution to converge to the continuous solution, namely, consistency. If $\boldsymbol{u}_{h}$ is replaced by $\boldsymbol{u}, p_{h}$ by $p$ and $\boldsymbol{\sigma}_{h}$ by $2 \nu \nabla^{S} \boldsymbol{u}$, it is easy to check that (80)-(82) hold. For this, it is crucial to have the boundary terms involving the pressure. 
The bilinear form of this problem is:

$$
\begin{aligned}
B_{\mathrm{SG}}\left(\left[\boldsymbol{u}_{h}, p_{h}, \boldsymbol{\sigma}_{h}\right],\left[\delta \boldsymbol{u}_{h}, \delta p_{h}, \delta \boldsymbol{\sigma}_{h}\right]\right) & =2 \nu\left(\nabla^{S} \boldsymbol{u}_{h}, \nabla^{S} \delta \boldsymbol{u}_{h}\right)_{\Omega}-\left(p_{h}, \nabla \cdot \delta \boldsymbol{u}_{h}\right)_{\Omega}-\left(\delta p_{h}, \nabla \cdot \boldsymbol{u}_{h}\right)_{\Omega} \\
& -\left\langle\boldsymbol{n} \cdot \boldsymbol{\sigma}_{h}, \delta \boldsymbol{u}_{h}\right\rangle_{\Gamma}-\left\langle\boldsymbol{n} \cdot \delta \boldsymbol{\sigma}_{h}, \boldsymbol{u}_{h}\right\rangle_{\Gamma}+\left\langle p_{h}, \boldsymbol{n} \cdot \delta \boldsymbol{u}_{h}\right\rangle_{\Gamma}+\left\langle\delta p_{h}, \boldsymbol{n} \cdot \boldsymbol{u}_{h}\right\rangle_{\Gamma} \\
& -\frac{1}{2 N_{0} \nu}\left(\delta \boldsymbol{\sigma}_{h}-2 \nu \nabla^{S} \delta \boldsymbol{u}_{h}, \boldsymbol{\sigma}_{h}-2 \nu \nabla^{S} \boldsymbol{u}_{h}\right)_{\Omega} .
\end{aligned}
$$

We shall prove that this bilinear form is stable in the norm

$$
\left\|\left[\boldsymbol{u}_{h}, p_{h}, \boldsymbol{\sigma}_{h}\right]\right\|_{\mathrm{SG}}^{2}=\nu\left\|\nabla \boldsymbol{u}_{h}\right\|_{L^{2}(\Omega)}^{2}+\frac{1}{\nu}\left\|p_{h}\right\|_{L^{2}(\Omega)}^{2}+\frac{\nu}{h}\left\|\boldsymbol{u}_{h}\right\|_{L^{2}(\Gamma)}^{2}+\frac{1}{\nu}\left\|\boldsymbol{\sigma}_{h}\right\|_{L^{2}(\Omega)}^{2} .
$$

In order to prove this, we shall need two conditions. The first is the vector counterpart of the compatibility condition (23)-(24), that will allow us to control the boundary term. It reads:

$$
\begin{aligned}
& \forall \boldsymbol{v}_{h} \in V_{h} \quad \exists \boldsymbol{\tau}_{h} \in \Sigma_{h}, \delta_{0}>0 \text { such that }\left\|\boldsymbol{v}_{h}\right\|_{L^{2}(\Gamma)}^{2} \lesssim\left\langle\boldsymbol{\tau}_{h} \cdot \boldsymbol{n}, \boldsymbol{v}_{h}\right\rangle_{\Gamma}+\delta_{0} h\left\|\nabla \boldsymbol{v}_{h}\right\|_{L^{2}(\Omega)}^{2}, \\
& \left\|\boldsymbol{\tau}_{h}\right\|_{L^{2}(\Gamma)}=\left\|\boldsymbol{v}_{h}\right\|_{L^{2}(\Gamma)}, \quad\left\|\boldsymbol{\tau}_{h}\right\|_{L^{2}(\Omega)}^{2} \lesssim h\left\|\boldsymbol{v}_{h}\right\|_{L^{2}(\Gamma)}^{2}
\end{aligned}
$$

Note that now $\Sigma_{h}$ is a space of symmetric tensors. Obviously, (85)-(86) hold under the same conditions as (23)(24).

The second condition is that the velocity-pressure pair $V_{h}-Q_{h}$ is inf-sup stable for the problem imposing the essential boundary conditions in a classical way on $\Omega_{h}$, that is to say,

$$
\forall q_{h} \in Q_{h} \quad \exists \boldsymbol{v}_{h} \in V_{h} \backslash\{\mathbf{0}\} \quad \text { such that } \quad \beta\left\|q_{h}\right\|_{L^{2}\left(\Omega_{h}\right)}\left\|\boldsymbol{v}_{h}\right\|_{H^{1}\left(\Omega_{h}\right)} \leq-\left(q_{h}, \nabla \cdot \boldsymbol{v}_{h}\right)_{\Omega_{h}},
$$

with $\beta>0$ and $\boldsymbol{v}_{h}$ vanishing on $\partial \Omega_{h}$. In the conforming approximation we use, $V_{h} \subset H^{1}\left(\Omega_{h}\right)^{d}$, Korn's inequality implies that $\left\|\nabla^{S} \boldsymbol{v}_{h}\right\|_{L^{2}\left(\Omega_{h}\right)}$ is a norm in $V_{h}$ equivalent to the $H^{1}\left(\Omega_{h}\right)$-norm.

Theorem 6. Assume that $N_{0}>1$ and that (85)-(86) and (87) hold. Then, if $h$ is sufficiently small, for all $\left[\boldsymbol{u}_{h}, p_{h}, \boldsymbol{\sigma}_{h}\right]$ there exists a non trivial $\left[\delta \boldsymbol{u}_{h}, \delta p_{h}, \delta \boldsymbol{\sigma}_{h}\right]$ such that:

$$
B_{\mathrm{SG}}\left(\left[\boldsymbol{u}_{h}, p_{h}, \boldsymbol{\sigma}_{h}\right],\left[\delta \boldsymbol{u}_{h}, \delta p_{h}, \delta \boldsymbol{\sigma}_{h}\right]\right) \gtrsim\left\|\left[\boldsymbol{u}_{h}, p_{h}, \boldsymbol{\sigma}_{h}\right]\right\|_{\mathrm{SG}}\left\|\left[\delta \boldsymbol{u}_{h}, \delta p_{h}, \delta \boldsymbol{\sigma}_{h}\right]\right\|_{\mathrm{SG}} .
$$

Proof. Similarly to the Poisson problem in Theorem 1, it can be shown that

$$
B_{\mathrm{SG}}\left(\left[\boldsymbol{u}_{h}, p_{h}, \boldsymbol{\sigma}_{h}\right],\left[\boldsymbol{u}_{h},-p_{h},-\boldsymbol{\sigma}_{h}-\beta_{1} h^{-1} \nu \tilde{\boldsymbol{\tau}}\right]\right) \gtrsim \nu\left\|\nabla \boldsymbol{u}_{h}\right\|_{L^{2}(\Omega)}^{2}+\frac{\nu}{h}\left\|\boldsymbol{u}_{h}\right\|_{L^{2}(\Gamma)}^{2}+\frac{1}{\nu}\left\|\boldsymbol{\sigma}_{h}\right\|_{L^{2}(\Omega)}^{2},
$$

where $\beta_{1}$ is an appropriate constant and $\tilde{\boldsymbol{\tau}}$ is the function that guarantees that (85)-(86) hold for $\boldsymbol{v}_{h}=\boldsymbol{u}_{h}$. In order to get control on the pressure, we make use of (87) for $q_{h}=p_{h}$. Let $\boldsymbol{v}_{h}^{p}$ be the element in $V_{h}$ for which (87) holds. Let us start noting that, since $\boldsymbol{v}_{h}^{p}=\mathbf{0}$ on $\partial \Omega_{h}$ :

$$
\left\|\boldsymbol{v}_{h}^{p}\right\|_{L^{2}(\Gamma)}^{2}=\sum_{K} \int_{K \cap \Gamma}\left|\boldsymbol{v}_{h}^{p}\right|^{2} \lesssim \sum_{K} \int_{K \cap \Gamma} h^{2}\left|\nabla \boldsymbol{v}_{h}^{p}\right|^{2} \lesssim h \sum_{K} \int_{K \cap \Omega_{\Gamma, \text { out }}}\left|\nabla \boldsymbol{v}_{h}^{p}\right|^{2} .
$$

On the other hand, since the measure of $\Omega_{\Gamma, \text { out }}$ tends to zero when $h \rightarrow 0$ and using standard inverse inequalities, it follows that

$$
-\left(p_{h}, \nabla \cdot \boldsymbol{v}_{h}^{p}\right)_{\Omega_{h}} \lesssim-\left(p_{h}, \nabla \cdot \boldsymbol{v}_{h}^{p}\right)_{\Omega}+\left\langle p_{h}, \boldsymbol{n} \cdot \boldsymbol{v}_{h}^{p}\right\rangle_{\Gamma}+\varphi(h)\left\|p_{h}\right\|_{L^{2}(\Omega)}\left\|\boldsymbol{v}_{h}^{p}\right\|_{H^{1}(\Omega)},
$$

where $\varphi(h) \rightarrow 0$ as $h \rightarrow 0$ and the last norms can be understood as normalizing factors. From (89) and (90) is follows that

$$
-\left(p_{h}, \nabla \cdot \boldsymbol{v}_{h}^{p}\right)_{\Omega}+\left\langle p_{h}, \boldsymbol{n} \cdot \boldsymbol{v}_{h}^{p}\right\rangle_{\Gamma} \gtrsim \frac{1}{\nu^{1 / 2}}\left\|p_{h}\right\|_{L^{2}(\Omega)} \nu^{1 / 2}\left(\left\|\nabla^{S} \boldsymbol{v}_{h}^{p}\right\|_{L^{2}(\Omega)}+\frac{1}{h^{1 / 2}}\left\|\boldsymbol{v}_{h}^{p}\right\|_{L^{2}(\Gamma)}\right),
$$

for $h$ small enough. Normalizing $\boldsymbol{v}_{h}^{p}$ so that

it is readily checked that

$$
\frac{1}{\nu^{1 / 2}}\left\|p_{h}\right\|_{L^{2}(\Omega)}=\nu^{1 / 2}\left(\left\|\nabla^{S} \boldsymbol{v}_{h}^{p}\right\|_{L^{2}(\Omega)}+\frac{1}{h^{1 / 2}}\left\|\boldsymbol{v}_{h}^{p}\right\|_{L^{2}(\Gamma)}\right),
$$

$$
B_{\mathrm{SG}}\left(\left[\boldsymbol{u}_{h}, p_{h}, \boldsymbol{\sigma}_{h}\right],\left[\boldsymbol{v}_{h}^{p}, 0, \mathbf{0}\right]\right) \gtrsim \frac{1}{\nu}\left\|p_{h}\right\|_{L^{2}(\Omega)}^{2}-\frac{1}{\nu}\left\|\boldsymbol{\sigma}_{h}\right\|_{L^{2}(\Omega)}^{2}-\nu\left\|\nabla^{S} \boldsymbol{u}_{h}\right\|_{L^{2}(\Omega)}^{2} .
$$

This, together with (88), allows us to prove the theorem using the same arguments as in the previous results. 


\subsubsection{Stabilized finite element approximation}

As for Darcy's problem, both in primal and dual forms, we can avoid the need of satisfying the inf-sup condition (87) by modifying the Galerkin finite element formulation and use instead a stabilized finite element method.

Assume for the sake of simplicity that $Q_{h}$ is made of continuous functions. The method we consider here is the following: find $\left[\boldsymbol{u}_{h}, p_{h}, \boldsymbol{\sigma}_{h}\right] \in V_{h} \times Q_{h} \times \Sigma_{h}$ such that

$$
B_{\mathrm{SS}}\left(\left[\boldsymbol{u}_{h}, p_{h}, \boldsymbol{\sigma}_{h}\right],\left[\delta \boldsymbol{u}_{h}, \delta p_{h}, \delta \boldsymbol{\sigma}_{h}\right]\right)=L_{\mathrm{SS}}\left(\left[\delta \boldsymbol{u}_{h}, \delta p_{h}, \delta \boldsymbol{\sigma}_{h}\right]\right), \quad \forall\left[\delta \boldsymbol{u}_{h}, \delta p_{h}, \delta \boldsymbol{\sigma}_{h}\right] \in V_{h} \times Q_{h} \times \Sigma_{h},
$$

where

$$
\begin{aligned}
B_{\mathrm{SS}}\left(\left[\boldsymbol{u}_{h}, p_{h}, \boldsymbol{\sigma}_{h}\right],\left[\delta \boldsymbol{u}_{h}, \delta p_{h}, \delta \boldsymbol{\sigma}_{h}\right]\right) & =B_{\mathrm{SG}}\left(\left[\boldsymbol{u}_{h}, p_{h}, \boldsymbol{\sigma}_{h}\right],\left[\delta \boldsymbol{u}_{h}, \delta p_{h}, \delta \boldsymbol{\sigma}_{h}\right]\right) \\
& +\sum_{K} \tau\left(2 \nu \nabla \cdot \nabla^{S} \delta \boldsymbol{u}_{h}+\nabla \delta p_{h},-2 \nu \nabla \cdot \nabla^{S} \boldsymbol{u}_{h}+\nabla p_{h}\right)_{K \cap \Omega}, \\
L_{\mathrm{SS}}\left(\left[\delta \boldsymbol{u}_{h}, \delta p_{h}, \delta \boldsymbol{\sigma}_{h}\right]\right) & =\left\langle\boldsymbol{f}, \delta \boldsymbol{u}_{h}\right\rangle_{\Omega}+\left\langle\delta p_{h}, \boldsymbol{n} \cdot \overline{\boldsymbol{u}}\right\rangle_{\Gamma}-\left\langle\boldsymbol{n} \cdot \delta \boldsymbol{\sigma}_{h}, \overline{\boldsymbol{u}}\right\rangle_{\Gamma} \\
& +\sum_{K} \tau\left(2 \nu \nabla \cdot \nabla^{S} \delta \boldsymbol{u}_{h}+\nabla \delta p_{h}, \boldsymbol{f}\right)_{K \cap \Omega}
\end{aligned}
$$

with

$$
\tau=\frac{h^{2}}{\nu} \tau^{0}
$$

and $\tau^{0}$ a dimensionless constant. See [1] and references therein for background about this method as well as for the treatment of discontinuous pressure interpolations.

If we define the norm

$$
\left\|\left[\boldsymbol{u}_{h}, p_{h}, \boldsymbol{\sigma}_{h}\right]\right\|_{\mathrm{SS}}^{2}=\nu\left\|\nabla \boldsymbol{u}_{h}\right\|_{L^{2}(\Omega)}^{2}+\frac{h^{2}}{\nu}\left\|\nabla p_{h}\right\|_{L^{2}(\Omega)}^{2}+\frac{\nu}{h}\left\|\boldsymbol{u}_{h}\right\|_{L^{2}(\Gamma)}^{2}+\frac{1}{\nu}\left\|\boldsymbol{\sigma}_{h}\right\|_{L^{2}(\Omega)}^{2},
$$

the following result is easily proved using the same techniques as for the previous theorems:

Theorem 7. Assume that $N_{0}>1$ and that (85)-(86) hold. Then, if $\tau^{0}$ in (93) is sufficiently small, for all $\left[\boldsymbol{u}_{h}, p_{h}, \boldsymbol{\sigma}_{h}\right]$ there exists a non trivial $\left[\delta \boldsymbol{u}_{h}, \delta p_{h}, \delta \boldsymbol{\sigma}_{h}\right]$ such that:

$$
B_{\mathrm{SS}}\left(\left[\boldsymbol{u}_{h}, p_{h}, \boldsymbol{\sigma}_{h}\right],\left[\delta \boldsymbol{u}_{h}, \delta p_{h}, \delta \boldsymbol{\sigma}_{h}\right]\right) \gtrsim\left\|\left[\boldsymbol{u}_{h}, p_{h}, \boldsymbol{\sigma}_{h}\right]\right\|_{\mathrm{SS}}\left\|\left[\delta \boldsymbol{u}_{h}, \delta p_{h}, \delta \boldsymbol{\sigma}_{h}\right]\right\|_{\mathrm{SS}} .
$$

Let us finally remark that $h^{2}\left\|\nabla p_{h}\right\|_{L^{2}(\Omega)}^{2}$ in (94) could be replaced by $\left\|p_{h}\right\|_{L^{2}(\Omega)}^{2}$ using the inf-sup condition that holds for the continuous problem, that is to say, the norm (84) could also be used in this case.

\subsection{Three-field approach}

To conclude the examples of elliptic problems where the methodology described in Section 2 can be applied, let us consider the three-field version of the Stokes problem for incompressible flows. The problem consists of finding the velocity $\boldsymbol{u}: \Omega \longrightarrow \mathbb{R}^{d}$, the pressure $p: \Omega \longrightarrow \mathbb{R}$ and the deviatoric stress $\boldsymbol{\tau}: \Omega \longrightarrow \mathbb{R}^{d \times d}$ solution of the boundary-value problem

$$
\begin{aligned}
\frac{1}{2 \nu} \boldsymbol{\tau}-\nabla^{S} \boldsymbol{u}=\mathbf{0} & \text { in } \Omega \\
-\nabla \cdot \boldsymbol{\tau}+\nabla p=\boldsymbol{f} & \text { in } \Omega \\
\nabla \cdot \boldsymbol{u}=0 & \text { in } \Omega \\
\boldsymbol{u}=\overline{\boldsymbol{u}} & \text { on } \Gamma
\end{aligned}
$$


Using the notation of Section 2, now we may identify $n=s+d+1, s$ being the number of components of symmetric tensors in $\mathbb{R}^{d \times d}, \mathcal{L}([\boldsymbol{\tau}, \boldsymbol{u}, p])=\left[\frac{1}{2 \nu} \boldsymbol{\tau}-\nabla^{S} \boldsymbol{u},-\nabla \cdot \boldsymbol{\tau}+\nabla p, \nabla \cdot \boldsymbol{u}\right], \mathcal{D}([\boldsymbol{\tau}, \boldsymbol{u}, p])=\boldsymbol{u}$ and

$$
\begin{aligned}
F([\boldsymbol{\eta}, \boldsymbol{v}, q]) & =-\frac{1}{4 \nu}\|\boldsymbol{\eta}\|_{L^{2}(\Omega)}^{2}+\left(\nabla^{S} \boldsymbol{v}, \boldsymbol{\eta}\right)_{\Omega}-(q, \nabla \cdot \boldsymbol{v})_{\Omega}-\langle\boldsymbol{f}, \boldsymbol{v}\rangle_{\Omega}, \\
B([\boldsymbol{\tau}, \boldsymbol{u}, p],[\delta \boldsymbol{\tau}, \delta \boldsymbol{u}, \delta p]) & =-\frac{1}{2 \nu}(\boldsymbol{\tau}, \delta \boldsymbol{\tau})_{\Omega}+\left(\nabla^{S} \boldsymbol{u}, \delta \boldsymbol{\tau}\right)_{\Omega}+\left(\nabla^{S} \delta \boldsymbol{u}, \boldsymbol{\tau}\right)_{\Omega}-(p, \nabla \cdot \delta \boldsymbol{u})_{\Omega}-(\delta p, \nabla \cdot \boldsymbol{u})_{\Omega}, \\
\mathcal{F}([\boldsymbol{\tau}, \boldsymbol{u}, p]) & =-p \boldsymbol{I}+\boldsymbol{\tau} .
\end{aligned}
$$

As for the two-field case, we now choose $N=N_{0} \nu$, with $N_{0}$ dimensionless. The problem is well posed in the space $X=T \times V \times Q=L^{2}(\Omega)_{\mathrm{sym}}^{d \times d} \times H^{1}(\Omega)^{d} \times L^{2}(\Omega) / \mathbb{R}$, where $L^{2}(\Omega)_{\mathrm{sym}}^{d \times d}$ is the space of symmetric second order tensors with square-integrable components and the space of traces is $\Lambda=H^{1 / 2}(\Gamma)^{d}$, the trace operator being $\left.[\boldsymbol{\tau}, \boldsymbol{u}, p] \mapsto \boldsymbol{u}\right|_{\Gamma}$.

\subsubsection{Galerkin finite element approximation}

Let $T_{h} \subset T, V_{h} \subset V$ and $Q_{h} \subset Q$ be the finite element spaces constructed from the finite element partition to approximate $T, V$ and $Q$, respectively, and let $\Sigma_{h}$ be the space of the new variable to be introduced for enforcing the boundary condition weakly. The functional to be optimized now is

$$
\hat{G}\left(\left[\boldsymbol{\eta}_{h}, \boldsymbol{v}_{h}, q_{h}, \boldsymbol{\xi}_{h}\right]\right)=F\left(\left[\boldsymbol{\eta}_{h}, \boldsymbol{v}_{h}, q_{h}\right]\right)-\left\langle\boldsymbol{n} \cdot \boldsymbol{\xi}_{h}, \boldsymbol{v}_{h}-\overline{\boldsymbol{u}}\right\rangle_{\Gamma}+\left\langle q_{h}, \boldsymbol{n} \cdot \boldsymbol{v}_{h}-\boldsymbol{n} \cdot \overline{\boldsymbol{u}}\right\rangle_{\Gamma}-\frac{1}{4 N_{0} \nu}\left\|\boldsymbol{\xi}_{h}-\boldsymbol{\eta}_{h}\right\|_{L^{2}(\Omega)}^{2},
$$

on $T_{h} \times V_{h} \times Q_{h} \times \Sigma_{h}$, and problem (14)-(15) in this case reads: find $\left[\boldsymbol{\tau}_{h}, \boldsymbol{u}_{h}, p_{h}, \boldsymbol{\sigma}_{h}\right] \in T_{h} \times V_{h} \times Q_{h} \times \Sigma_{h}$ such that

$$
\begin{aligned}
-\frac{1}{2 \nu}\left(\boldsymbol{\tau}_{h}, \delta \boldsymbol{\tau}_{h}\right)_{\Omega}+\left(\nabla^{S} \boldsymbol{u}_{h}, \delta \boldsymbol{\tau}_{h}\right)_{\Omega}-\frac{1}{2 N_{0} \nu}\left(-\delta \boldsymbol{\tau}_{h}, \boldsymbol{\sigma}_{h}-\boldsymbol{\tau}_{h}\right)_{\Omega} & =\mathbf{0} \\
\left(\nabla^{S} \delta \boldsymbol{u}_{h}, \boldsymbol{\tau}_{h}\right)_{\Omega}-\left(p_{h}, \nabla \cdot \delta \boldsymbol{u}_{h}\right)_{\Omega}-\left\langle\boldsymbol{n} \cdot \boldsymbol{\sigma}_{h}, \delta \boldsymbol{u}_{h}\right\rangle_{\Gamma}+\left\langle p_{h}, \boldsymbol{n} \cdot \delta \boldsymbol{u}_{h}\right\rangle_{\Gamma} & =\left\langle\boldsymbol{f}, \delta \boldsymbol{u}_{h}\right\rangle_{\Omega}, \\
-\left(\delta p_{h}, \nabla \cdot \boldsymbol{u}_{h}\right)_{\Omega}+\left\langle\delta p_{h}, \boldsymbol{n} \cdot \boldsymbol{u}_{h}\right\rangle_{\Gamma} & =\left\langle\delta p_{h}, \boldsymbol{n} \cdot \overline{\boldsymbol{u}}\right\rangle_{\Gamma}, \\
-\left\langle\boldsymbol{n} \cdot \delta \boldsymbol{\sigma}_{h}, \boldsymbol{u}_{h}\right\rangle_{\Gamma}-\frac{1}{2 N_{0} \nu}\left(\delta \boldsymbol{\sigma}_{h}, \boldsymbol{\sigma}_{h}-\boldsymbol{\tau}_{h}\right)_{\Omega} & =-\left\langle\boldsymbol{n} \cdot \delta \boldsymbol{\sigma}_{h}, \overline{\boldsymbol{u}}\right\rangle_{\Gamma},
\end{aligned}
$$

for all $\left[\delta \boldsymbol{\tau}_{h}, \delta \boldsymbol{u}_{h}, \delta p_{h}, \delta \boldsymbol{\sigma}_{h}\right] \in T_{h} \times V_{h} \times Q_{h} \times \Sigma_{h}$. The bilinear form of this problem is:

$$
\begin{aligned}
B_{\mathrm{S} 3 \mathrm{G}}\left(\left[\boldsymbol{\tau}_{h}, \boldsymbol{u}_{h}, p_{h}, \boldsymbol{\sigma}_{h}\right],\left[\delta \boldsymbol{\tau}_{h}, \delta \boldsymbol{u}_{h}, \delta p_{h}, \delta \boldsymbol{\sigma}_{h}\right]\right) & \left.=-\frac{1}{2 \nu}\left(\boldsymbol{\tau}_{h}, \delta \boldsymbol{\tau}_{h}\right)_{\Omega}+\left(\nabla^{S} \boldsymbol{u}_{h}, \delta \boldsymbol{\tau}_{h}\right)_{\Omega}+(\nabla)^{S} \delta \boldsymbol{u}_{h}, \boldsymbol{\tau}_{h}\right)_{\Omega} \\
& -\left(\delta p_{h}, \nabla \cdot \boldsymbol{u}_{h}\right)_{\Omega}-\left(p_{h}, \nabla \cdot \delta \boldsymbol{u}_{h}\right)_{\Omega}-\left\langle\boldsymbol{n} \cdot \boldsymbol{\sigma}_{h}, \delta \boldsymbol{u}_{h}\right\rangle_{\Gamma} \\
& -\left\langle\boldsymbol{n} \cdot \delta \boldsymbol{\sigma}_{h}, \boldsymbol{u}_{h}\right\rangle_{\Gamma}+\left\langle p_{h}, \boldsymbol{n} \cdot \delta \boldsymbol{u}_{h}\right\rangle_{\Gamma}+\left\langle\delta p_{h}, \boldsymbol{n} \cdot \boldsymbol{u}_{h}\right\rangle_{\Gamma} \\
& -\frac{1}{2 N_{0} \nu}\left(\delta \boldsymbol{\sigma}_{h}-\delta \boldsymbol{\tau}_{h}, \boldsymbol{\sigma}_{h}-\boldsymbol{\tau}_{h}\right)_{\Omega} .
\end{aligned}
$$

It turns out that the norm in which $B_{S 3 G}$ is stable is

$$
\left\|\left[\boldsymbol{\tau}_{h}, \boldsymbol{u}_{h}, p_{h}, \boldsymbol{\sigma}_{h}\right]\right\|_{\mathrm{S} 3 \mathrm{G}}^{2}=\nu\left\|\nabla \boldsymbol{u}_{h}\right\|_{L^{2}(\Omega)}^{2}+\frac{1}{\nu}\left\|p_{h}\right\|_{L^{2}(\Omega)}^{2}+\frac{1}{\nu}\left\|\boldsymbol{\tau}_{h}\right\|_{L^{2}(\Omega)}^{2}+\frac{\nu}{h}\left\|\boldsymbol{u}_{h}\right\|_{L^{2}(\Gamma)}^{2}+\frac{1}{\nu}\left\|\boldsymbol{\sigma}_{h}\right\|_{L^{2}(\Omega)}^{2} .
$$

In order to prove that this, we shall need two conditions. The first is again the compatibility condition between $V_{h}$ and $\Sigma_{h}$ encountered for the two-field formulation, i.e., (85)-(86). The second condition is that the stress-velocitypressure combination $T_{h}-V_{h}-Q_{h}$ is inf-sup stable for the problem imposing the essential boundary conditions in a classical way on $\Omega_{h}$, that is to say, condition (87) holds as well as

$$
\forall \boldsymbol{v}_{h} \in V_{h} \quad \exists \boldsymbol{\eta}_{h} \in T_{h} \backslash\{\mathbf{0}\} \quad \text { such that } \beta\left\|\boldsymbol{v}_{h}\right\|_{H^{1}\left(\Omega_{h}\right)}\left\|\boldsymbol{\eta}_{h}\right\|_{L^{2}\left(\Omega_{h}\right)} \leq\left(\nabla^{S} \boldsymbol{v}_{h}, \boldsymbol{\eta}_{h}\right)_{\Omega_{h}},
$$

with $\beta>0$. There are a few triplets $T_{h}-V_{h}-Q_{h}$ known to satisfy (87)-(95) (see references and comments in [7]).

Theorem 8. Assume that $N_{0}>1$ and that (85)-(86) and (87)-(95) hold. Then, if h is sufficiently small, for all $\left[\boldsymbol{\tau}_{h}, \boldsymbol{u}_{h}, p_{h}, \boldsymbol{\sigma}_{h}\right]$ there exists a non trivial $\left[\delta \boldsymbol{\tau}_{h}, \delta \boldsymbol{u}_{h}, \delta p_{h}, \delta \boldsymbol{\sigma}_{h}\right]$ such that:

$$
B_{\mathrm{S} 3 \mathrm{G}}\left(\left[\boldsymbol{u}_{h}, p_{h}, \boldsymbol{\sigma}_{h}\right],\left[\delta \boldsymbol{u}_{h}, \delta p_{h}, \delta \boldsymbol{\sigma}_{h}\right]\right) \gtrsim\left\|\left[\boldsymbol{u}_{h}, p_{h}, \boldsymbol{\sigma}_{h}\right]\right\|_{\mathrm{S} 3 \mathrm{G}}\left\|\left[\delta \boldsymbol{u}_{h}, \delta p_{h}, \delta \boldsymbol{\sigma}_{h}\right]\right\|_{\mathrm{S} 3 \mathrm{G}} .
$$


The proof uses a combination of the techniques employed in Theorem 2 for Darcy's problem in primal form and in Theorem 6 for the two-field Stokes problem, in both cases using the Galerkin finite element approximation.

\subsubsection{Stabilized finite element approximation}

Finally, we can also consider a stabilized finite element method for the three-field Stokes problem. When boundary conditions are imposed in the classical way, the method we will consider is similar to the one proposed and analyzed in [7], obtained by replacing the orthogonal projection of this reference by the identity applied to finite element residuals. It allows one to avoid the need of satisfying (87)-(95).

Assume for the sake of simplicity that $T_{h}$ and $Q_{h}$ are made of continuous functions. The formulation we propose is the following: find $\left[\boldsymbol{\tau}_{h}, \boldsymbol{u}_{h}, p_{h}, \boldsymbol{\sigma}_{h}\right] \in T_{h} \times V_{h} \times Q_{h} \times \Sigma_{h}$ such that

$$
B_{\mathrm{S} 3 \mathrm{~S}}\left(\left[\boldsymbol{\tau}_{h}, \boldsymbol{u}_{h}, p_{h}, \boldsymbol{\sigma}_{h}\right],\left[\delta \boldsymbol{\tau}_{h}, \delta \boldsymbol{u}_{h}, \delta p_{h}, \delta \boldsymbol{\sigma}_{h}\right]\right)=L_{\mathrm{S} 3 \mathrm{~S}}\left(\left[\delta \boldsymbol{\tau}_{h}, \delta \boldsymbol{u}_{h}, \delta p_{h}, \delta \boldsymbol{\sigma}_{h}\right]\right),
$$

for all $\left[\delta \boldsymbol{\tau}_{h}, \delta \boldsymbol{u}_{h}, \delta p_{h}, \delta \boldsymbol{\sigma}_{h}\right] \in T_{h} \times V_{h} \times Q_{h} \times \Sigma_{h}$, where

$$
\begin{aligned}
B_{\mathrm{S} 3 \mathrm{~S}}\left(\left[\boldsymbol{u}_{h}, p_{h}, \boldsymbol{\sigma}_{h}\right],\left[\delta \boldsymbol{u}_{h}, \delta p_{h}, \delta \boldsymbol{\sigma}_{h}\right]\right) & =B_{\mathrm{S} 3 \mathrm{G}}\left(\left[\boldsymbol{u}_{h}, p_{h}, \boldsymbol{\sigma}_{h}\right],\left[\delta \boldsymbol{u}_{h}, \delta p_{h}, \delta \boldsymbol{\sigma}_{h}\right]\right) \\
& +\sum_{K} \tau_{T}\left(-\frac{1}{2 \nu} \delta \boldsymbol{\tau}_{h}-\nabla^{S} \delta \boldsymbol{u}_{h}, \frac{1}{2 \nu} \boldsymbol{\tau}_{h}-\nabla^{S} \boldsymbol{u}_{h}\right)_{K \cap \Omega} \\
& +\sum_{K} \tau_{V}\left(-\nabla \cdot \delta \boldsymbol{\tau}_{h}+\nabla \delta p_{h},-\nabla \cdot \boldsymbol{\tau}_{h}+\nabla p_{h}\right)_{K \cap \Omega} \\
L_{\mathrm{S} 3 \mathrm{~S}}\left(\left[\delta \boldsymbol{u}_{h}, \delta p_{h}, \delta \boldsymbol{\sigma}_{h}\right]\right) & =\left\langle\boldsymbol{f}, \delta \boldsymbol{u}_{h}\right\rangle_{\Omega}+\left\langle\delta p_{h}, \boldsymbol{n} \cdot \overline{\boldsymbol{u}}\right\rangle_{\Gamma}-\left\langle\boldsymbol{n} \cdot \delta \boldsymbol{\sigma}_{h}, \overline{\boldsymbol{u}}\right\rangle_{\Gamma} \\
& +\sum_{K} \tau_{V}\left(-\nabla \cdot \delta \boldsymbol{\tau}_{h}+\nabla \delta p_{h}, \boldsymbol{f}\right)_{K \cap \Omega},
\end{aligned}
$$

with

$$
\tau_{T}=2 \nu \tau_{T}^{0}, \quad \tau_{V}=\frac{h^{2}}{\nu} \tau_{V}^{0}
$$

and $\tau_{T}^{0}$ and $\tau_{V}^{0}$ are dimensionless constants.

If we define the norm

$$
\left\|\left[\boldsymbol{\tau}_{h}, \boldsymbol{u}_{h}, p_{h}, \boldsymbol{\sigma}_{h}\right]\right\|_{\mathrm{S} 3 \mathrm{~S}}^{2}=\nu\left\|\nabla \boldsymbol{u}_{h}\right\|_{L^{2}(\Omega)}^{2}+\frac{h^{2}}{\nu}\left\|\nabla p_{h}\right\|_{L^{2}(\Omega)}^{2}+\frac{1}{\nu}\left\|\boldsymbol{\tau}_{h}\right\|_{L^{2}(\Omega)}^{2}+\frac{\nu}{h}\left\|\boldsymbol{u}_{h}\right\|_{L^{2}(\Gamma)}^{2}+\frac{1}{\nu}\left\|\boldsymbol{\sigma}_{h}\right\|_{L^{2}(\Omega)}^{2},
$$

we have that

Theorem 9. Assume that $N_{0}>1$ and that (85)-(86) hold. Then, if in (96) $\tau_{V}^{0}$ is sufficiently small and $0<\tau_{T}^{0}<1$, for all $\left[\boldsymbol{\tau}_{h}, \boldsymbol{u}_{h}, p_{h}, \boldsymbol{\sigma}_{h}\right]$ there exists a non trivial $\left[\delta \boldsymbol{\tau}_{h}, \delta \boldsymbol{u}_{h}, \delta p_{h}, \delta \boldsymbol{\sigma}_{h}\right]$ such that:

$$
B_{\mathrm{S} 3 \mathrm{~S}}\left(\left[\boldsymbol{\tau}_{h}, \boldsymbol{u}_{h}, p_{h}, \boldsymbol{\sigma}_{h}\right],\left[\delta \boldsymbol{\tau}_{h}, \delta \boldsymbol{u}_{h}, \delta p_{h}, \delta \boldsymbol{\sigma}_{h}\right]\right) \gtrsim\left\|\left[\boldsymbol{\tau}_{h}, \boldsymbol{u}_{h}, p_{h}, \boldsymbol{\sigma}_{h}\right]\right\|_{\mathrm{S} 3 \mathrm{~S}}\left\|\left[\delta \boldsymbol{\tau}_{h}, \delta \boldsymbol{u}_{h}, \delta p_{h}, \delta \boldsymbol{\sigma}_{h}\right]\right\|_{\mathrm{S} 3 \mathrm{~S}} .
$$

In this case, the proof uses a combination of the techniques employed in Theorem 3 for Darcy's problem in primal form and in Theorem 7 for the two-field Stokes problem, in both cases using a stabilized finite element approximation.

\section{Numerical examples}

In this section we show some numerical examples which illustrate the performance of the proposed methods for weakly imposing essential boundary conditions in non-matching meshes. The numerical examples are applied to the Poisson problem, the stabilized mixed form of Darcy's problem, and the stabilized two-field Stokes problem. 


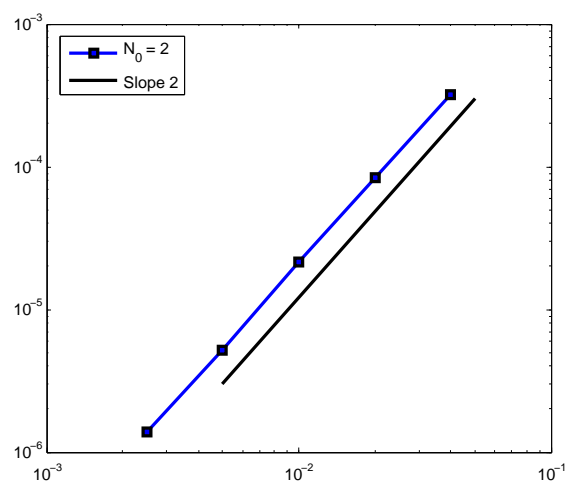

Figure 2: Convergence plot for Poisson's problem.
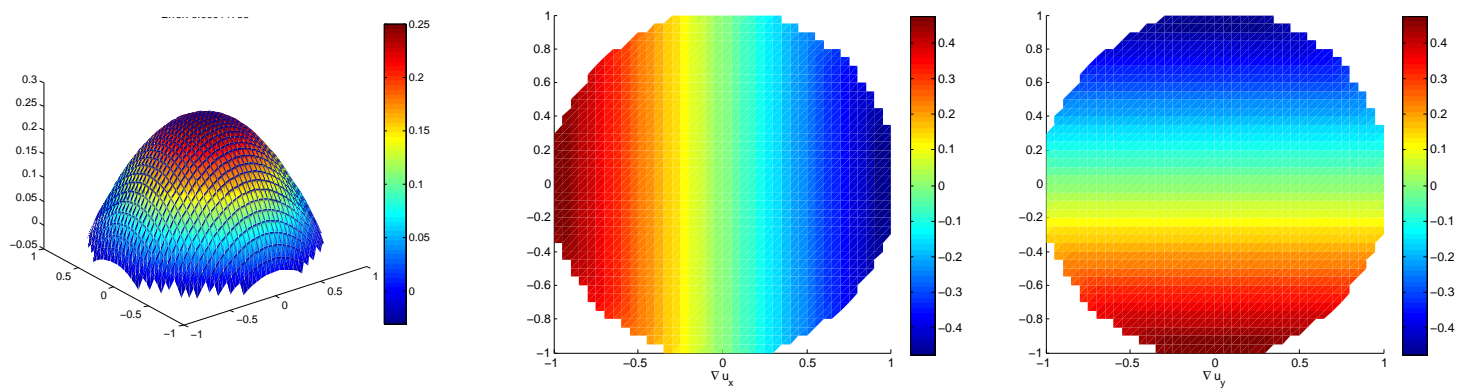

Figure 3: Solution field $u_{h}$ and its gradient $\nabla u_{h, x}, \nabla u_{h, y}$.

\subsection{Poisson's Problem}

For the Poisson problem, we solve the heat equation in a circle of radius 1 . The external domain over which the finite element mesh extends is the $[1,1] \times[-1,1]$ square. A structured linear triangle mesh is used in this domain and we look for the solution to Poisson's problems with unit diffusion coefficient and uniform unitary source term. The unknown is set to zero in the boundary of the domain. The space for the Lagrange multipliers $\Sigma_{h}$ is taken to be piecewise constant and discontinuous across interelement boundaries. The analytical solution to this problem is $u(x, y)=\frac{1}{4}\left(R^{2}-x^{2}-y^{2}\right)$.

Fig. 2 shows the errors $\left\|u-u_{h}\right\|_{L^{2}(\Omega)}$ versus the element size $h$, for the proposed method. It can be concluded from the convergence curve that optimal (quadratic) convergence for Poisson's problem is obtained when linear elements are used. In Fig. 3 the solution field and its gradient are presented. Finally, Fig. shows the difference between the solution gradient $k \nabla u_{h}$ and the Lagrange multipliers $\boldsymbol{\sigma}_{h}$. It can be observed that in interior elements they coincide. In elements which are cut by the boundary the values for the Lagrange multipliers differ from the values for the gradients. The largest differences are observed in elements in which the interior volume is small with respect to the length of the element boundary. This agrees with what is expected from equation (21).

\subsection{Darcy's problem}

In this numerical example we test the proposed method for the stabilized, mixed form of Darcy's problem. The analyzed problem is the same as in the Poisson problem, but in this case we introduce an additional variable for the fluxes. Both the temperature $u_{h}$ and the fluxes $\boldsymbol{q}_{h}$ fields are approximated using triangular linear finite elements. Again the space for the Lagrange multipliers $\Sigma_{h}$ is taken elementwise discontinuous, but in this case the interpolation space is linear. As in the previous example, this choice for $\Sigma_{h}$ allows to condensate the Lagrange 

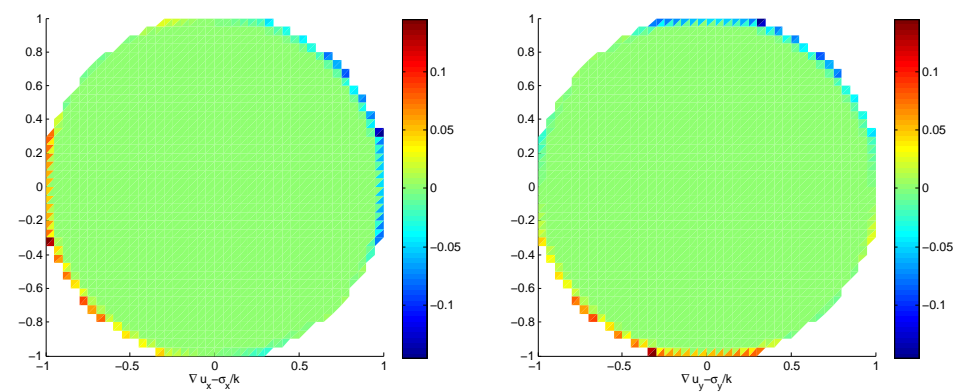

Figure 4: Difference between $k \nabla u_{h}$ and the Lagrange multipliers $\boldsymbol{\sigma}_{h}$ for the Poisson problem.
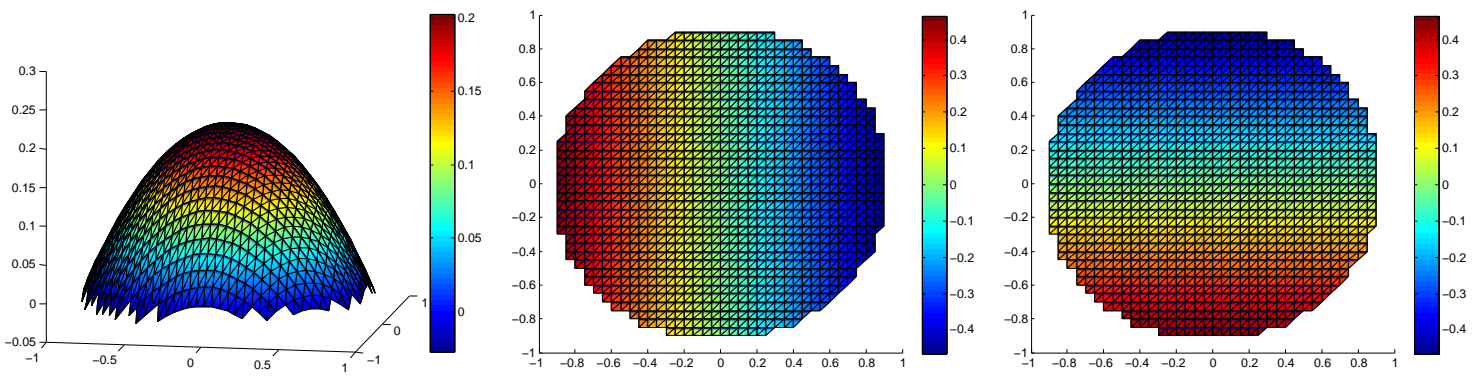

Figure 5: $u_{h}, q_{x, h}$ and $q_{y, h}$ plots for the solution of the stabilized Darcy mixed problem.

multipliers and end up with a problem written only in terms of $u_{h}$ and $\boldsymbol{q}_{h}$. Fig. 5 shows the $u_{h}$ and $\boldsymbol{q}_{h}$ solution fields, which are very similar to the ones obtained for the Poisson problem. The convergence plot for several mesh sizes is shown in Fig. 6. It can be observed that $u_{h}$ converges quadratically, while the convergence for $\boldsymbol{q}_{h}$ is second order for coarse meshes but linear as the mesh is refined. This agrees with the expected convergence rates for the stabilized Darcy problem in mixed form.

In Fig. 7 we plot the difference between the fluxes $\boldsymbol{q}_{h}$ and the Lagrange multipliers $\boldsymbol{\sigma}_{h}$ for the primal form of Darcy's problem. The difference is null in interior elements, and the largest differences are again found in elements which have a small interior volume compared to the size of the element boundary.

\subsection{Stokes' Problem}

This numerical example involves the solution of the stationary Stokes flow past a cylinder. Linear interpolations are used for both the velocity and the pressure fields, but the Lagrange multipliers are approximated as piecewise discontinuous. The setting of the problem is shown in Fig. 8 . A parabolic inflow profile with unitary mean horizontal velocity is set on $x=0$. Velocity is prescribed to zero on $y=0$ and $y=1$ and on the cylindric boundary.

Fig. 9 shows the resulting velocity and pressure fields. In Fig. 10 the error versus the mesh size is plotted, both for the velocity and for the pressure fields. Results for each mesh size have been compared against results in a much finer mesh (160000 elements). Quadratic convergence rates are obtained in both cases. Finally, the gradient of the velocity field $k \nabla \boldsymbol{u}_{h}$ is shown in Fig. 11 together with the difference between this gradient and the stress Lagrange multipliers $\sigma_{h}$. As in the previous examples, they are exactly equivalent everywhere except in the boundary elements, where maximum differences are observed in elements with a large surface/interior volume ratio. 


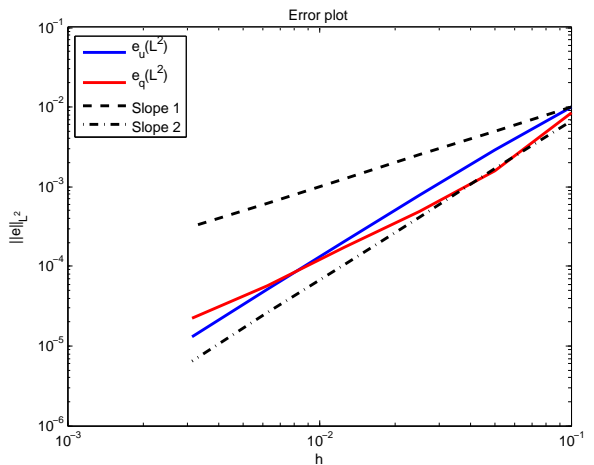

Figure 6: Convergence for Darcy's problem in mixed form (equal interpolation).
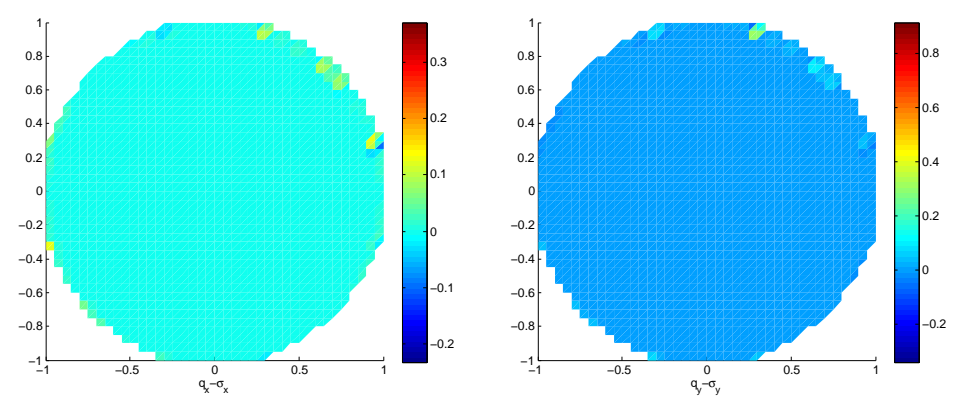

Figure 7: Difference between the fluxes $\boldsymbol{q}_{h}$ and the Lagrange multipliers $\boldsymbol{\sigma}_{h}$ for the primal form of Darcy's problem.

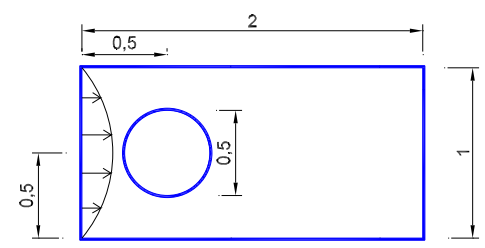

Figure 8: Setting for the Stokes problem. 

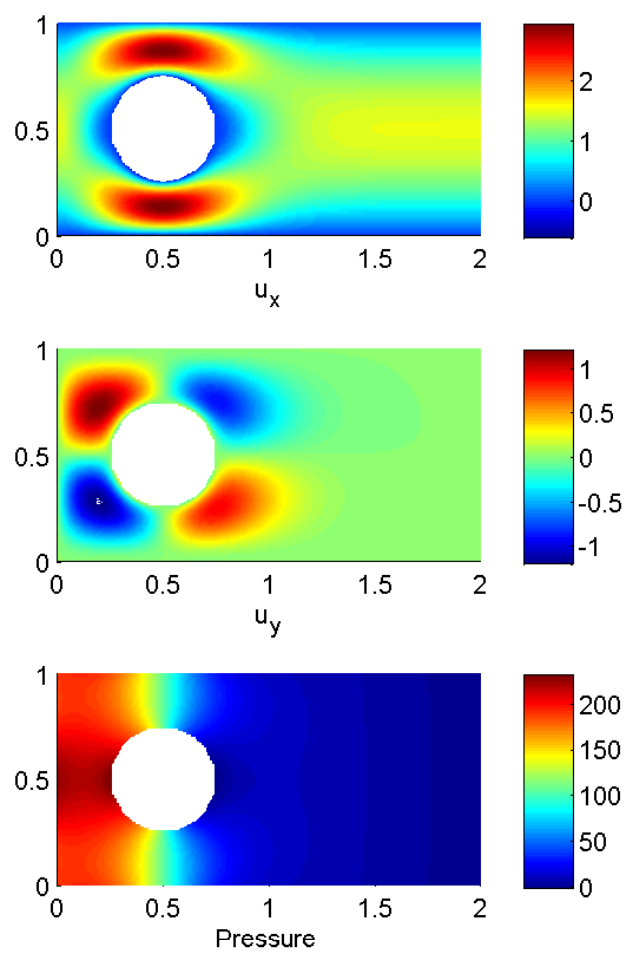

Figure 9: Velocity and pressure fields for the Stokes problem.

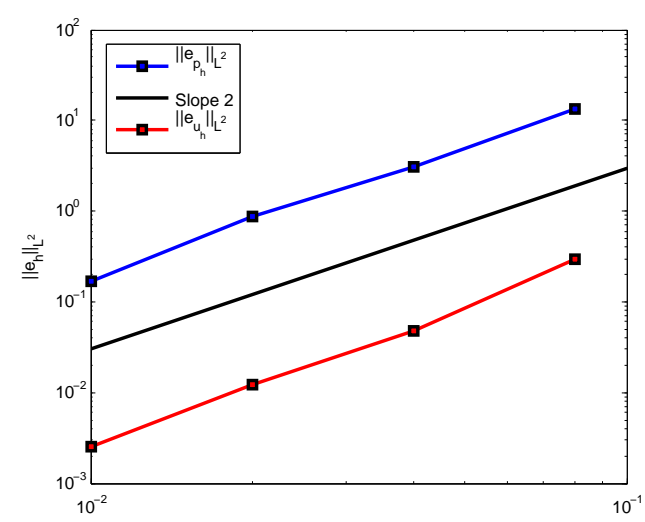

Figure 10: Convergence plot for the Stokes problem. 

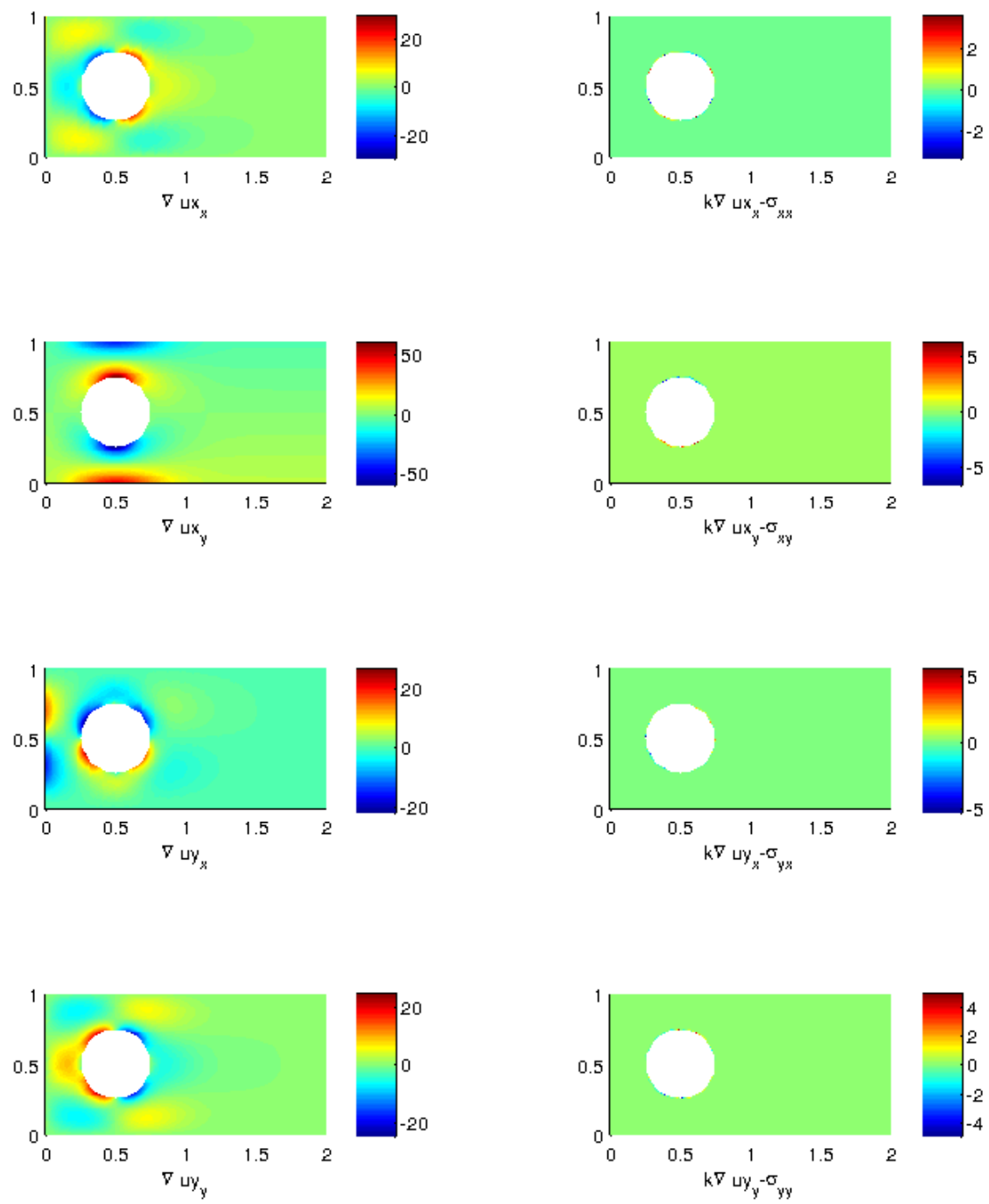

Figure 11: Velocity gradient $k \nabla \boldsymbol{u}_{h}$ and difference between the velocity gradient and the Lagrange multiplier field $\sigma_{h}$ for the Stokes problem. 


\section{Conclusions}

In this paper we have presented a general methodology for imposing essential boundary conditions in finite element approximations where the boundary of the computational domain does not match the element boundaries. The method can be described starting from the classical Lagrange multiplier technique, but then relating the Lagrange multiplier to the flux of the problem in a least-squares sense. It is therefore variationally founded. Except for the Darcy problem in dual form, the new variable can be condensed at the element level. In all cases, it is defined on the whole computational domain (or maybe just close to the boundary), and not only on the boundary where essential boundary conditions need to be imposed. The final method resembles also Nitsche's methods, but the penalty parameter needs not to be very large.

We have analyzed the stability of the method in a variety of elliptic problems. We have tried to stress the stability requirements for the new field introduced, as well as to show how do they interact with the conditions for stability of the original problem being solved. In the case of the mixed methods treated, both the cases of inf-sup stable interpolations and of stabilized finite element methods have been studied. Altogether, we have presented nine stability results, two of which were already presented in [4]. Other elliptic problems, such as the elasticity equations or Maxwell's problem, could be treated with the same technique to prescribe boundary conditions.

Numerical experiments have shown that the method proposed works well, and that it displays optimal order of convergence, although we have preferred not to analyze convergence in this work. Even if we have motivated it from the optimization of a functional associated to an elliptic problem, the method as such can be applied to other type of problems. The study of its performance in more general situations, particularly in flow problems, was already started in [4] and deserves further research. In particular, it can be an alternative to Nitsche's method to prescribe Dirichlet conditions in a way less stringent than incorporating them in the approximation space.

\section{Acknowledgments}

R. Codina acknowledges the support received from the ICREA Acadèmia Program, from the Catalan Government. J. Baiges acknowledges the support received from the Spanish Government through a Juan de la Cierva postdoctoral grant.

\section{References}

[1] S. Badia and R. Codina. Unified stabilized finite element formulations for the Stokes and the Darcy problems. SIAM Journal on Numerical Analysis, 47:1971-2000, 2009.

[2] S. Badia and R. Codina. Stokes, Maxwell and Darcy: a single finite element approximation for three model problems. Applied Numerical Mathematics, 62:246-263, 2012.

[3] J. Baiges and R. Codina. The fixed-mesh ALE approach applied to solid mechanics and fluid-structure interaction problems. International Journal for Numerical Methods in Engineering, 81:1529-1557, 2010.

[4] J. Baiges, R. Codina, F. Henke, S. Shahmiri, and W.A. Wall. A symmetric method for weakly imposing Dirichlet boundary conditions in embedded finite element meshes. International Journal for Numerical Methods in Engineering, 90:636-658, 2012.

[5] F. Brezzi and M. Fortin. Mixed and hybrid finite element methods. Springer Verlag, 1991.

[6] R. Codina. Stabilization of incompressibility and convection through orthogonal sub-scales in finite element methods. Computer Methods in Applied Mechanics and Engineering, 190:1579-1599, 2000.

[7] R. Codina. Finite element approximation of the three field formulation of the Stokes problem using arbitrary interpolations. SIAM Journal on Numerical Analysis, 47:699-718, 2009.

[8] R. Codina and J. Baiges. Approximate imposition of boundary conditions in immersed boundary methods. International Journal for Numerical Methods in Engineering, 80:1379-1405, 2009.

[9] R. Codina and J. Baiges. Fixed Mesh Methods in Computational Mechanics, in Developments and Applications in Engineering Computational Technology, B.H.V. Topping, J.M. Adam, F.J. Pallars, R. Bru and M.L. Romero (Eds.), chapter 4, pages 81-102. Saxe-Coburg Publications, 2010. 
[10] R. Codina and G. Houzeaux. Implementation aspects of coupled problems in CFD involving time dependent domains, in Verification and Validation Methods for Challenging Multiphysics Problems, G. Bugeda, J.C Courty, A. Guilliot, R. Höld, M. Marini, T. Nguyen, K. Papailiou, J. Périaux and D. Schwamborn (Eds.), pages 99-123. CIMNE, Barcelona, 2006.

[11] R. Codina, J. Houzeaux, H. Coppola-Owen, and J. Baiges. The fixed-mesh ALE approach for the numerical approximation of flows in moving domains. Journal of Computational Physics, 228:1591-1611, 2009.

[12] H. Coppola-Owen and R. Codina. A finite element model for free surface flows on fixed meshes. International Journal for Numerical Methods in Fluids, 54:1151-1171, 2007.

[13] J. Donea, A. Huerta, J.-Ph. Ponthot, and A. Rodríguez-Ferran. Arbitrary Lagrangian-Eulerian Methods, in Encyclopedia of Computational Mechanics. Volume 1: Fundamentals., E. Stein, R. de Borst and T.J.R. Hughes (Eds.), chapter 14. John Wiley \& Sons, 2004.

[14] A. Gerstenberger and W.A. Wall. An embedded Dirichlet formulation for 3D continua. International Journal for Numerical Methods in Engineering, 82:537-563, 2010.

[15] A. Gilmanov and F. Sotiropoulos. A hybrid Cartesian/immersed boundary method for simulating flows with 3D, geometrically complex, moving bodies. Journal of Computational Physics, 207:457-492, 2005.

[16] R. Glowinski, T.-W. Pan, and J. Périaux. A fictitious domain method for Dirichlet problems and applications. Computer Methods in Applied Mechanics and Engineering, 111:203-303, 1994.

[17] R. Glowinski, T.W. Pan, T.I. Hesla, D.D. Joseph, and J. Périaux. A distributed Lagrange multiplier/fictitious domain method for flows around moving rigid bodies: application to particulate flow. International Journal for Numerical Methods in Fluids, 30:1043-1066, 1999.

[18] M. Juntunen and R. Stenberg. Nitsche's method for general boundary conditions. Mathematics of Computation, 78:1353-1374, 2009.

[19] Ming-Chih Lai and C.S. Peskin. An immersed boundary method with formal second-order accuracy and reduced numerical viscosity. Journal of Computational Physics, 160:705-719, 2000.

[20] R.J. LeVeque and Z. Li. The immersed interface method for elliptic equations with discontinuous coefficients and singular sources. SIAM Journal on Numerical Analysis, 31 (4):1019-1044, 1994.

[21] R.J. LeVeque and Z. Li. Immersed interface method for incompressible Navier-Stokes equations. SIAM Journal on Scientific and Statistical Computing, 18 (3):709-735, 1997.

[22] J. Mohd-Yusof. Combined immersed boundaries/B-splines methods for simulations of flows in complex geometries. CTR annual research briefs, Stanford University, NASA Ames, 1997.

[23] C.S. Peskin. Flow patterns around heart valves: A numerical method. Journal of Computational Physics, 10:252-271, 1972.

[24] J.V. Voorde, J. Vierendeels, and E. Dick. Flow simulations in rotary volumetric pumps and compressors with the fictitious domain method. Journal of Computational and Applied Mathematics, 168:491-499, 2004.

[25] S. Xu and Z.J. Wang. An immersed interface method for simulating the interaction of a fluid with moving boundaries. Journal of Computational Physics, 216:454-493, 2006.

[26] J.H. Ferziger Y.H. Tseng. A ghost-cell immersed boundary method for flow in complex geometry. Journal of Computational Physics, 192:593-623, 2003. 